\title{
Summability of multilinear mappings: Littlewood, Orlicz and beyond
}

\author{
Oscar Blasco*, Geraldo Botelho, Daniel Pellegrino ${ }^{\dagger}$ and Pilar Rueda ${ }^{\ddagger}$
}

\begin{abstract}
In this paper we prove a plenty of new results concerning summabililty properties of multilinear mappings between Banach spaces, such as an extension of Littlewood's $4 / 3$ Theorem. Among other features, it is shown that every continuous $n$-linear form on the disc algebra $\mathcal{A}$ or the Hardy space $\mathcal{H}^{\infty}$ is $(1 ; 2, \ldots, 2)$-summing, the role of the Littlewood-Orlicz property in the theory is established and the interplay with almost summing multilinear mappings is explored.
\end{abstract}

\section{Introduction}

Motivated by several matters related to linear functional analysis, such as integral equations, Fourier analysis and analytic number theory, the theory of multilinear forms and polynomials on Banach spaces was initiated in the beginning of the last century with the works of several outstanding mathematicians like Banach, Bohr, Bohnenblust, Hille, Littlewood, Orlicz, Schur, etc. In 1930, Littlewood [27] proved a celebrated theorem asserting that

$$
\left(\sum_{i, j=1}^{\infty}\left|A\left(e_{i}, e_{j}\right)\right|^{\frac{4}{3}}\right)^{\frac{3}{4}} \leq \sqrt{2}\|A\|
$$

for every continuous bilinear form $A$ on $c_{0} \times c_{0}$. One year later, Bohnenblust and Hille [6] realized the importance of this result to the convergence of ordinary Dirichlet series and extended Littlewood's result to multilinear mappings in the following fashion:

If $A$ is a continuous $n$-linear form on $c_{0} \times \cdots \times c_{0}$, then there is a constant $C_{n}$ (depending only on $n$ ) such that

$$
\left(\sum_{i_{1}, \ldots, i_{n}=1}^{\infty} \mid A\left(e_{i_{1}}, \ldots, e_{i_{n}}\right)^{\frac{2 n}{n+1}}\right)^{\frac{n+1}{2 n}} \leq C_{n}\|A\|
$$

These results can be regarded as the beginning of the study of summability properties of multilinear mappings between Banach spaces. This line of investigation has been developed since then and more recently it has found its place within the theory of ideals of

\footnotetext{
*Supported by MEC and FEDER Projects MTM2005-08350-C03-03 and MTM2008-04594/MTM.

${ }^{\dagger}$ Supported by CNPq Project 471054/2006-2 and Grant 308084/2006-3.

${ }^{\ddagger}$ Supported by MEC and FEDER Project MTM2005-08210.

2000 Mathematics Subject Classification. Primary 46G25; Secondary 47B10.

Keywords: absolutely summing multilinear mapping, type and cotype, Littlewood Theorem.
} 
multilinear mappings outlined by Pietsch [36] in 1983. In this context classes of absolutely summing multilinear mappings are studied as generalizations of the very successful theory of absolutely summing linear operators. The theory has been successfully developed by several authors (a list of references is omitted because it would grow very large) and even applications to Quantum Mechanics have been recently found (see [35]). One of the trends of the theory of absolutely summing multilinear mappings is the search for summability properties in the spirit of those of Littlewood's and Bohnenblust-Hille's theorems (see, e.g., [2, 10, 13, 17, 28, 33, 34]). In this paper we aim to give new contributions to this line of investigation in several directions, which we describe next.

Two well known results related to the linear theory are Grothendieck's theorem, that asserts that every continuous linear operator from $\ell_{1}$ to $\ell_{2}$ is absolutely summing, and the weak Dvoretzky-Rogers theorem, that asserts that the identity operator of any infinite dimensional Banach space fails to be absolutely $p$-summing for any $1 \leq p<\infty$. These two important results can be considered as the roots of what has been known as coincidence and non-coincidence results. The passage from the linear to the multilinear case has occasioned the emergence of several coincidence and non-coincidence situations for absolutely summing multilinear mappings (see [1, 17, 11, 12, 13, 30, 31, 32, 33, 34]). The scope of the present paper is to prove new coincidence theorems, some of them generalizing known results and some giving new perspectives to the subject.

Respecting the historical development of the subject we start in Section 2 by extending the classical Littlewood $4 / 3$-Theorem by proving that, given $1 \leq p \leq 2$ and $\frac{1}{q}=\frac{1}{2}+\frac{1}{p^{\prime}}$, any continuous bilinear functional $A$ defined on $c_{0} \times c_{0}$ satisfies that $\left(A\left(e_{j}, e_{k}\right)\right)_{j k}$ belongs to $\ell_{p}\left(\ell_{q}\right)$, where $\left(e_{j}\right)_{j}$ is the unit basis. Actually we prove a more general version of this result, in which by taking $p=4 / 3$ we recover Littlewood's theorem. In Section 3 we prove coincidence and inclusion theorems that will be useful in later sections. While the role of the Orlicz property in the theory is well established, in Section 4 we show that the Littlewood-Orlicz property can be used to get even stronger results. More precisely, we prove that for suitable $n, p_{1}, p_{2}, \ldots, p_{n}$, any continuous $n$-linear mapping defined on a product of Banach spaces, one of which has a dual with the Littlewood-Orlicz property, is absolutely $\left(p ; p_{1}, \ldots, p_{n}\right)$-summing. We also generalize a coincidence result due to Pérez-García (see Theorem 4.3). Inspired by this generalization, in Section 5 we develop a general technique of extending bilinear coincidences to $n$-linear coincidences, $n \geq 3$. In Section [ 6] almost summing operators are used to get some more summability properties of multilinear mappings. Calling on the type/cotype theory we get for instance that for any $1 \leq p \leq 2$, every continuous bilinear functional $A$ defined on $\ell_{p} \times F$, where $F$ is a Banach space whose dual has type 2 , is absolutely $(p ; 2,1)$-summing. Moreover, if $1 \leq p \leq 2$ then $A$ is absolutely $\left(r_{p} ; r_{p}, r_{p}\right)$-summing for any $1 \leq r_{p} \leq \frac{2 p}{3 p-2}$.

\section{Notation and background}

Henceforth $E_{1}, \ldots, E_{n}, E, F$ will be Banach spaces over the scalar field $\mathbb{K}=\mathbb{R}$ or $\mathbb{C}, B_{E}$ represents the closed unit ball of $E$ and the topological dual of $E$ will be denoted by $E^{\prime}$. The Banach space of all continuous $n$-linear mappings from $E_{1} \times \cdots \times E_{n}$ into $F$ is denoted by $\mathcal{L}\left(E_{1}, \ldots, E_{n} ; F\right)$. As usual we write $\mathcal{L}\left({ }^{n} E ; F\right)$ if $E_{1}=\cdots=E_{n}=E$. For the general theory of polynomials/multilinear mappings between Banach spaces we refer to [22, 29].

Let $p>0$. By $\ell_{p}(E)$ we denote the Banach space of all absolutely $p$-summable sequences $\left(x_{j}\right)_{j=1}^{\infty}$ in $E$ endowed with its usual $\ell_{p}$-norm $(p$-norm if $0<p<1)$. Let $\ell_{p}^{w}(E)$ be the space of those sequences $\left(x_{j}\right)_{j=1}^{\infty}$ in $E$ such that $\left(\varphi\left(x_{j}\right)\right)_{j=1}^{\infty} \in \ell_{p}$ for every $\varphi \in E^{\prime}$ 
endowed with the norm $(p$-norm if $0<p<1)$

$$
\left\|\left(x_{j}\right)_{j=1}^{\infty}\right\|_{\ell_{p}^{w}(E)}=\sup _{\varphi \in B_{E^{\prime}}}\left(\sum_{j=1}^{\infty}\left|\varphi\left(x_{j}\right)\right|^{p}\right)^{\frac{1}{p}} .
$$

Let $\ell_{p}^{u}(E)$ denote the closed subspace of $\ell_{p}^{w}(E)$ formed by the sequences $\left(x_{j}\right)_{j=1}^{\infty} \in \ell_{p}^{w}(E)$ such that $\lim _{k \rightarrow \infty}\left\|\left(x_{j}\right)_{j=k}^{\infty}\right\|_{\ell_{p}^{w}(E)}=0$.

Let $A \in \mathcal{L}\left(E_{1}, \ldots, E_{n} ; F\right)$ and let $X_{1} \ldots, X_{n}, Y$ be spaces of sequences in $E_{1}, \ldots, E_{n}, F$ respectively. Whenever we say that $\hat{A}: X_{1} \times \cdots \times X_{n} \longrightarrow Y$ is bounded we mean that the correspondence

$$
\begin{gathered}
\left(\left(x_{j}^{1}\right)_{j=1}^{\infty}, \ldots,\left(x_{j}^{n}\right)_{j=1}^{\infty}\right) \in X_{1} \times \cdots \times X_{n} \mapsto \\
\hat{A}\left(\left(x_{j}^{1}\right)_{j=1}^{\infty}, \ldots,\left(x_{j}^{n}\right)_{j=1}^{\infty}\right):=\left(A\left(x_{j}^{1}, \ldots, x_{j}^{n}\right)\right)_{j=1}^{\infty} \in Y
\end{gathered}
$$

is well defined into $Y$ (hence multilinear) and continuous.

For $0<p, p_{1}, p_{2}, \ldots, p_{n} \leq \infty$, we assume that $\frac{1}{p} \leq \frac{1}{p_{1}}+\cdots+\frac{1}{p_{n}}$. A multilinear mapping $A \in \mathcal{L}\left(E_{1}, \ldots, E_{n} ; F\right)$ is absolutely $\left(p ; p_{1}, p_{2}, \ldots, p_{n}\right)$-summing if there exists $C>0$ such that

$$
\left\|\left(A\left(x_{j}^{1}, x_{j}^{2}, \ldots, x_{j}^{n}\right)\right)_{j}\right\|_{p} \leq C \prod_{i=1}^{n}\left\|\left(x_{j}^{i}\right)_{j}\right\|_{\ell_{p_{i}}^{w}\left(E_{i}\right)}
$$

for all finite family of vectors $x_{j}^{i}$ in $E_{i}$ for $i=1,2, \ldots, n$. The infimum of such $C>0$ is called the $\left(p ; p_{1}, \ldots, p_{n}\right)$-summing norm of $A$ and is denoted by $\pi_{\left(p ; p_{1}, \ldots, p_{n}\right)}(A)$. Let $\Pi_{\left(p ; p_{1}, p_{2}, \ldots, p_{n}\right)}\left(E_{1}, \ldots, E_{n} ; F\right)$ denote the space of all absolutely $\left(p ; p_{1}, p_{2}, \ldots, p_{n}\right)$-summing $n$-linear mappings from $E_{1} \times \cdots \times E_{n}$ to $F$ endowed with the norm $\pi_{\left(p ; p_{1} \ldots, p_{n}\right)}$. Thus, $A \in \Pi_{\left(p ; p_{1}, p_{2}, \ldots, p_{n}\right)}\left(E_{1}, \ldots, E_{n} ; F\right)$ if and only if

$$
\hat{A}: \ell_{p_{1}}^{w}\left(E_{1}\right) \times \ell_{p_{2}}^{w}\left(E_{2}\right) \times \cdots \times \ell_{p_{n}}^{w}\left(E_{n}\right) \rightarrow \ell_{p}(F) \text { is bounded } .
$$

It is well known that we can replace $\ell_{p_{k}}^{w}\left(E_{k}\right)$ by $\ell_{p_{k}}^{u}\left(E_{k}\right)$ in the definition of absolutely summing mappings.

Absolutely $\left(\frac{p}{n} ; p, \ldots, p\right)$-summing $n$-linear mappings are usually called $p$-dominated. They satisfy the following factorization result (see [36, Theorem 13]):

$A \in \Pi_{\left(\frac{p}{n} ; p, \ldots, p\right)}\left(E_{1}, \ldots, E_{n} ; F\right)$ if and only if there are Banach spaces $G_{1}, \ldots, G_{n}$, operators $u_{j} \in \Pi_{p}\left(E_{j} ; G_{j}\right)$ and $B \in \mathcal{L}\left(G_{1}, \ldots, G_{n} ; F\right)$ such that

$$
A=B \circ\left(u_{1}, \ldots, u_{n}\right) \text {. }
$$

Let us now recall some basic facts about Rademacher functions and its use in Banach space theory. For each $1 \leq p \leq \infty$, we denote by $\operatorname{Rad}_{p}(E)$ the space of sequences $\left(x_{j}\right)_{j=1}^{\infty}$ in $E$ such that

$$
\left\|\left(x_{j}\right)_{j=1}^{\infty}\right\|_{\operatorname{Rad}_{p}(E)}=\sup _{n \in \mathbb{N}}\left\|\sum_{j=1}^{n} r_{j} x_{j}\right\|_{L^{p}([0,1], E)}<\infty,
$$

where $\left(r_{j}\right)_{j \in \mathbb{N}}$ are the Rademacher functions on $[0,1]$ defined by $r_{j}(t)=\operatorname{sign}\left(\sin 2^{j} \pi t\right)$. The reader is referred to [21, 41, 42] for the difference between this space and the space of sequences $\left(x_{n}\right)$ for which the series $\sum_{n=1}^{\infty} x_{n} r_{n}$ is convergent in $L^{p}([0,1], E)$. It is easy to see that $\operatorname{Rad}_{\infty}(E)$ coincides with $\ell_{1}^{w}(E)$. Making use of the Kahane's inequalities (see [21, p. 211]) it follows that the spaces $\operatorname{Rad}_{p}(E)$ coincide up to equivalent norms for all $1 \leq p<\infty$. The unique vector space so obtained will therefore be denoted by $\operatorname{Rad}(E)$, and we agree to (mostly) use the norm $\|\cdot\|_{\operatorname{Rad}(E)}:=\|\cdot\|_{\operatorname{Rad}_{2}(E)}$ on $\operatorname{Rad}(E)$. 
Recall also that a linear operator $u: E \rightarrow F$ is said to be almost summing if there is a $C>0$ such that we have

$$
\left\|\left(u\left(x_{j}\right)\right)_{j=1}^{m}\right\|_{\operatorname{Rad}(F)} \leq C\left\|\left(x_{j}\right)_{j=1}^{m}\right\|_{\ell_{2}^{w}(E)}
$$

for any finite set of vectors $\left\{x_{1}, \ldots, x_{m}\right\}$ in $E$. The space of all almost summing linear operators from $E$ to $F$ is denoted by $\Pi_{a . s}(E ; F)$ and the infimum of all $C>0$ fulfilling the above inequality is denoted by $\|u\|_{a . s}$. Note that this definition differs from the definition of almost summing operators given in [21, p. 234] but coincides with the characterization which appears a few lines after that definition (yes, the definition and the stated characterization are not equivalent). Since the proof of [21, Proposition 12.5] uses the characterization (which is our definition) we can conclude that every absolutely $p$-summing linear operator, $1 \leq p<+\infty$, is almost summing.

The concept of almost summing multilinear mappings was considered in [8, 9] as reads as follows: A multilinear map $A \in \mathcal{L}\left(E_{1}, \ldots, E_{n} ; F\right)$ is said to be almost summing if there exists $C>0$ such that we have

$$
\left\|\left(A\left(x_{j}^{1}, \ldots, x_{j}^{n}\right)\right)_{j=1}^{m}\right\|_{R a d(F)} \leq C \prod_{i=1}^{n}\left\|\left(x_{j}^{i}\right)_{j=1}^{m}\right\|_{\ell_{2}^{w}\left(E_{i}\right)}
$$

for any finite set of vectors $\left(x_{j}^{i}\right)_{j=1}^{m} \subset E_{i}$ for $i=1, \ldots, n$. We write $\Pi_{a . s}\left(E_{1}, \ldots, E_{n} ; F\right)$ for the space of almost summing multilinear maps, which is endowed with the norm

$$
\|A\|_{a s}:=\inf \{C>0 \text { such that (2) holds }\} .
$$

For the theory of type and cotype in Banach spaces the reader is referred to [21, Chapter 11]. Recall that a Banach space $E$ is said to have the Orlicz property if there exists a constant $C>0$ such that

$$
\left(\sum_{j=1}^{n}\left\|x_{j}\right\|^{2}\right)^{1 / 2} \leq C \sup _{t \in[0,1]}\left\|\sum_{j=1}^{n} x_{j} r_{j}(t)\right\|
$$

for any finite family $x_{1}, x_{2}, \ldots x_{n}$ of vectors in $E$. In other words, $E$ has the Orlicz property when the identity operator $i d_{E}$ is absolutely $(2 ; 1)$-summing.

One should notice that, due to results by Talagrand (see [39, 40]), while the Orlicz property is weaker than cotype 2 , having cotype $q>2$ is equivalent to the existence of a constant $C>0$ such that

$$
\left(\sum_{j=1}^{n}\left\|x_{j}\right\|^{q}\right)^{1 / q} \leq C \sup _{t \in[0,1]}\left\|\sum_{j=1}^{n} x_{j} r_{j}(t)\right\|
$$

for any finite family $x_{1}, x_{2}, \ldots x_{n}$ of vectors in $E$.

A relevant property for our purposes is the following: We say that a Banach space $E$ has the Littlewood-Orlicz property if $\ell_{1}^{w}(E)$ is continuously contained in the projective tensor product $\ell_{2} \otimes_{\pi} E$ (for a related concept of Littlewood-Orlicz operator we refer to [16, Section 4]). Of course, since $\ell_{2} \otimes_{\pi} E \subset \ell_{2}(E)$, the Littlewood-Orlicz property implies Orlicz-property. 
In [18], J. S. Cohen introduces the space

$$
\ell_{p}\langle E\rangle:=\left\{\left(x_{n}\right)_{n=1}^{\infty} \subset E: \sum_{n=1}^{\infty}\left|x_{n}^{*}\left(x_{n}\right)\right|<\infty \text { for each }\left(x_{n}^{*}\right)_{n=1}^{\infty} \in \ell_{p^{\prime}}^{w}\left(E^{\prime}\right)\right\},
$$

where $1 / p+1 / p^{\prime}=1$ and the space of operators $p$-Cohen-nuclear $u \in \mathcal{L}(E, F)$ such that

$$
\left\|\left(u\left(x_{j}\right)\right)_{j=1}^{m}\right\|_{l_{p}\langle F\rangle} \leq C\left\|\left(x_{j}\right)_{j=1}^{m}\right\|_{\ell_{p}^{w}(E)}
$$

for all finite family of vectors $x_{1}, x_{2}, \ldots, x_{m}$ in $E$.

It was first shown that $\ell_{p} \otimes_{\pi} E \subset \ell_{p}\langle E\rangle$ (see [18, Theorem 1.1.3 (i)]) and, later the space $\ell_{p}\langle E\rangle$ was shown to coincide with $\ell_{p} \otimes_{\pi} E$ (see [15, Theorem 1] or [4]) for $1<p<\infty$.

The reader is referred to [3] for a description in terms of integral operators, where $\ell_{p}\langle E\rangle$ is denoted $\ell_{\pi_{1, p^{\prime}}}(E)$, and for a proof of $\ell_{2}\langle E\rangle \subset \operatorname{Rad}(E)$. Therefore we always have

$$
\left.\left(\ell_{2} \otimes_{\pi} E\right)\right) \cap \ell_{1}^{w}(E) \subset \operatorname{Rad}(E) \subset \ell_{2}^{w}(E) .
$$

The following result was obtained in [3, Theorem 9]:

$$
\ell_{2} \otimes_{\pi} E=\operatorname{Rad}(E) \Longleftrightarrow E \text { is a GT-space of cotype } 2
$$

where $E$ being a $G T$-space means that every continuous linear mapping from $E$ to $\ell_{2}$ is absolutely 1-summing. In particular every GT-space with cotype 2 has the LittlewoodOrlicz property. The basic examples are $\mathcal{L}_{1}$-spaces and other examples of GT-spaces with cotype 2 can be found in [37.

Let us end this preliminary section by mentioning that the complex interpolation method, for which the reader is referred to [5, Theorem 5.1.2] or [41, Theorem 3.1], and a complexification technique (see [33, Section IV.2]) will be applied several times in Section 5. The complexification technique will allow us reduce proofs to the complex case. Similar applications of this interpolation-complexification argument can be found in [10, 24, 33.

\section{An extension of Littlewood's $4 / 3$ theorem}

Littlewood 27] proved that if $A: c_{0} \times c_{0} \rightarrow \mathbb{K}$ is a continuous bilinear form, then

$$
\left(\sum_{j, k}\left|A\left(e_{j}, e_{k}\right)\right|^{4 / 3}\right)^{3 / 4} \leq c\|A\|
$$

with $c=\sqrt{2}$. It is well-known that the constant $c=\sqrt{2}$ is far from being optimal, for example in [19, Theorem 34.11] or [41, Theorem 11.11] it is proved that in the complex case the best constant $c$ satisfying (5) is dominated by $2^{1 / 4} K_{G}^{1 / 2}$, i.e.,

$$
c \leq 2^{1 / 4} K_{G}^{1 / 2}
$$

where $K_{G}$ is Grothendieck's constant (note that $2^{1 / 4} K_{G}^{1 / 2}<\sqrt{2}$ in the complex case since $K_{G}<\sqrt{2}$ in this case). To the best of our knowledge the best estimate known for this constant is $c \leq K_{G}$ [26, Corollary 2, p. 280].

In this section we extend Littlewood's Theorem in the complex case to a more general setting in which the estimate for the best constant remains $K_{G}$, that is, we improve the result keeping the best known constant. 
Given a matrix $m_{j k}$ we write

$$
\left\|\left(m_{j k}\right)\right\|_{\ell_{p}\left(\ell_{q}\right)}=\left(\sum_{k}\left(\sum_{j}\left|m_{j k}\right|^{q}\right)^{p / q}\right)^{1 / p} .
$$

If $a$ and $\beta$ are matrices, we denote by $(\beta \circ a)_{j k}$ the product of $\beta$ and $a$, that is

$$
(\beta \circ a)_{j k}=\sum_{l} \beta_{j l} a_{l k}
$$

Theorem 2.1. Let $A \in \mathcal{L}\left({ }^{2} c_{0} ; \mathbb{C}\right)$. If $a=\left(a_{j k}\right)_{j, k}:=\left(A\left(e_{j}, e_{k}\right)\right)_{j, k}, 1 \leq p \leq 2$ and $\frac{1}{q}=\frac{1}{2}+\frac{1}{p^{\prime}}$, then

$$
\left\|(\beta \circ a)_{j k}\right\|_{\ell_{p}\left(\ell_{q}\right)} \leq K_{G}\|A\|\left\|\left(\beta_{j k}\right)\right\|_{\ell_{\infty}\left(\ell_{2}\right)}
$$

that is

$$
\left(\sum_{k}\left(\sum_{j}\left|\sum_{l} \beta_{j l} A\left(e_{l}, e_{k}\right)\right|^{q}\right)^{p / q}\right)^{1 / p} \leq K_{G}\|A\| \sup _{k}\left(\sum_{j}\left|\beta_{j k}\right|^{2}\right)^{1 / 2} .
$$

In particular, selecting $\beta$ as the identity matrix,

$$
\left(\sum_{k}\left(\sum_{j}\left|A\left(e_{j}, e_{k}\right)\right|^{q}\right)^{p / q}\right)^{1 / p} \leq K_{G}\|A\| .
$$

Selecting $p=4 / 3$ we recover Littlewood's Theorem, that is $\left(A\left(e_{j}, e_{k}\right)\right)_{j k} \in \ell_{4 / 3}\left(\mathbb{N}^{2}\right)$.

Proof. From [34, Corollary 2.5] we know that

$$
\sum_{j}\left|A\left(y_{j}, x_{j}\right)\right| \leq K_{G}\|A\|\left\|\left(x_{j}\right)\right\|_{\ell_{2}^{w}\left(c_{0}\right)}\left\|\left(y_{j}\right)\right\|_{\ell_{2}^{w}\left(c_{0}\right)} .
$$

Now write $x_{j}(k)=\lambda_{j k}$ and $y_{j}(k)=\beta_{j k}$. Since the canonical basis of $\ell_{1}$ is a norming set of $c_{0}$, from [21, p. 36] we know that

$$
\left\|\left(x_{j}\right)\right\|_{\ell_{2}^{w}\left(c_{0}\right)}=\sup _{k}\left(\sum_{j}\left|\lambda_{j k}\right|^{2}\right)^{1 / 2} \text { and }\left\|\left(y_{j}\right)\right\|_{\ell_{2}^{w}\left(c_{0}\right)}=\sup _{k}\left(\sum_{j}\left|\beta_{j k}\right|^{2}\right)^{1 / 2} .
$$

So, we have

$$
\left|\sum_{j} \sum_{k, l} \lambda_{j k} \varepsilon_{j} \beta_{j l} A\left(e_{l}, e_{k}\right)\right| \leq K_{G}\|A\| \sup _{k}\left(\sum_{j}\left|\lambda_{j k}\right|^{2}\right)^{1 / 2} \sup _{l}\left(\sum_{j}\left|\beta_{j l}\right|^{2}\right)^{1 / 2}
$$

for a convenient choice of $\varepsilon_{j} \in \mathbb{C}$ with $\left|\varepsilon_{j}\right|=1$. Note that

$$
\left(\lambda_{j k}\right)=\left(\left(\lambda_{j k}\right)_{j=1}^{\infty}\right)_{k=1}^{\infty} \in \ell_{\infty}\left(\ell_{2}\right) \text { and }\left(\beta_{j l}\right)=\left(\left(\beta_{j l}\right)_{j=1}^{\infty}\right)_{l=1}^{\infty} \in \ell_{\infty}\left(\ell_{2}\right)
$$

Hence

$$
\left|\sum_{j} \sum_{k, l} \lambda_{j k} \varepsilon_{j} \beta_{j l} A\left(e_{l}, e_{k}\right)\right| \leq K_{G}\|A\|\left\|\left(\lambda_{j k}\right)\right\|_{\ell_{\infty}\left(\ell_{2}\right)}\left\|\left(\beta_{j l}\right)\right\|_{\ell_{\infty}\left(\ell_{2}\right)} .
$$

Using the duality $\left(\ell_{1}\left(\ell_{2}\right)\right)^{*}=\ell_{\infty}\left(\ell_{2}\right)$ and the inequality

$$
\left|\sum_{j, k}\left(\sum_{l} \beta_{j l} \varepsilon_{j} A\left(e_{l}, e_{k}\right)\right) \lambda_{j k}\right| \leq K_{G}\|A\|\left\|\left(\lambda_{j k}\right)\right\|_{\ell_{\infty}\left(\ell_{2}\right)}\left\|\left(\beta_{j l}\right)\right\|_{\ell_{\infty}\left(\ell_{2}\right)}
$$


one obtains that

$$
\left(\sum_{l} \beta_{j l} \varepsilon_{j} A\left(e_{l}, e_{k}\right)\right)_{j k}=\left(\left(\sum_{l} \beta_{j l} \varepsilon_{j} A\left(e_{l}, e_{k}\right)\right)_{j=1}^{\infty}\right)_{k=1}^{\infty} \in \ell_{1}\left(\ell_{2}\right),
$$

and also

$$
\sum_{k}\left(\sum_{j}\left|\left(\sum_{l} \beta_{j l} \varepsilon_{j} A\left(e_{l}, e_{k}\right)\right)\right|^{2}\right)^{1 / 2} \leq K_{G}\|A\|\left\|\left(\beta_{j l}\right)\right\|_{\ell_{\infty}\left(\ell_{2}\right)} .
$$

Hence

$$
\sum_{k}\left(\sum_{j}\left|\sum_{l} \beta_{j l} A\left(e_{l}, e_{k}\right)\right|^{2}\right)^{1 / 2} \leq K_{G}\|A\|\left\|\left(\beta_{j l}\right)\right\|_{\ell_{\infty}\left(\ell_{2}\right)} .
$$

But from [23, Corollary 5.4.2 with $p=1$ and $q=2]$ we know that

$$
\left(\sum_{k}\left(\sum_{j}\left|\sum_{l} \beta_{j l} A\left(e_{l}, e_{k}\right)\right|\right)^{2}\right)^{1 / 2} \leq \sum_{k}\left(\sum_{j}\left|\sum_{l} \beta_{j l} A\left(e_{l}, e_{k}\right)\right|^{2}\right)^{1 / 2} .
$$

It follows that

$$
\begin{aligned}
\left\|(\beta \circ a)_{j k}\right\|_{\ell_{1}\left(\ell_{2}\right)} & \leq K_{G}\|A\|\left\|\left(\beta_{j k}\right)\right\|_{\ell_{\infty}\left(\ell_{2}\right)} \text { and } \\
\left\|(\beta \circ a)_{j k}\right\|_{\ell_{2}\left(\ell_{1}\right)} & \leq K_{G}\|A\|\left\|\left(\beta_{j k}\right)\right\|_{\ell_{\infty}\left(\ell_{2}\right) .}
\end{aligned}
$$

Now complex interpolation gives that

$$
\left(\ell_{1}\left(\ell_{2}\right), \ell_{2}\left(\ell_{1}\right)\right)_{[\theta]}=\ell_{p}\left(\left(\ell_{2}, \ell_{1}\right)_{[\theta]}\right)
$$

with

$$
\frac{1}{p}=(1-\theta)+\frac{\theta}{2}
$$

One concludes that

$$
\left(\ell_{1}\left(\ell_{2}\right), \ell_{2}\left(\ell_{1}\right)\right)_{[\theta]}=\ell_{p}\left(\ell_{q}\right)
$$

with

$$
\left.\frac{1}{p}=(1-\theta)+\frac{\theta}{2} \text { and } \frac{1}{q}=\frac{1-\theta}{2}+\theta \text { (hence } \frac{1}{q}=\frac{1}{2}+\frac{1}{p^{\prime}}\right) .
$$

So $(\beta \circ a)_{j k} \in \ell_{p}\left(\ell_{q}\right)$ and

$$
\left\|(\beta \circ a)_{j k}\right\|_{\ell_{p}\left(\ell_{q}\right)} \leq K_{G}\|A\|\left\|\left(\beta_{j k}\right)\right\|_{\ell_{\infty}\left(\ell_{2}\right)}
$$

that is

$$
\left(\sum_{k}\left(\sum_{j}\left|\sum_{l} \beta_{j l} A\left(e_{l}, e_{k}\right)\right|^{q}\right)^{p / q}\right)^{1 / p} \leq K_{G}\|A\| \sup _{k}\left(\sum_{j}\left|\beta_{j k}\right|^{2}\right)^{1 / 2} .
$$

Finally note that $\theta=1 / 2$ gives $p=4 / 3$ and $q=4 / 3$.

Remark 2.2. As pointed out before, although our result holds in a more general setting, the estimate $K_{G}$ for the best Littlewood constant we have just obtained in the complex case improves the estimate $2^{1 / 4} K_{G}^{1 / 2}$ given in [19, Theorem 34.11] and [41, Theorem 11.11] and equals the best known estimate.

Remark 2.3. Making $p=1$ and $q=2$ we recover the so-called general Littlewood inequality that appears in [26, (2.10), p. 280]. 


\section{Some general coincidence results}

Defant-Voigt Theorem (see [1, Theorem 3.10]) stating that

$$
\mathcal{L}\left(E_{1}, \ldots, E_{n} ; \mathbb{K}\right)=\Pi_{(1 ; 1, \ldots, 1)}\left(E_{1}, \ldots, E_{n} ; \mathbb{K}\right)
$$

is probably the first and most folkloric coincidence result in the theory of absolutely summing multilinear mappings. The next result gives a slightly more general version.

Proposition 3.1. Let $n \geq 2$ and $A \in \mathcal{L}\left(E_{1}, \ldots, E_{n} ; \mathbb{K}\right)$. Then

$$
\hat{A}: \operatorname{Rad}\left(E_{1}\right) \times \cdots \times \operatorname{Rad}\left(E_{n}\right) \rightarrow \ell_{1}
$$

is bounded. Moreover $\|\hat{A}\|=\|A\|$.

Proof. Let $\left(x_{j}^{i}\right)$ be finite sequences in $E_{i}$ for $i=1, \ldots, n$. One can find a sequence $\left(\alpha_{j}\right)$ of norm one scalars so that

$$
\sum_{j}\left|A\left(x_{j}^{1}, \ldots, x_{j}^{n}\right)\right|=\sum_{j} A\left(\alpha_{j} x_{j}^{1}, \ldots, x_{j}^{n}\right) .
$$

Let

$$
\begin{aligned}
f_{\alpha}\left(t_{1}\right) & =\sum_{j} \alpha_{j} r_{j}\left(t_{1}\right) x_{j}^{i}, \\
f_{i}\left(t_{i}\right) & =\sum_{j} r_{j}\left(t_{i}\right) x_{j}^{i}, i=2, \ldots, n-1, \text { and } \\
f_{n}\left(t_{1}, \ldots, t_{n-1}\right) & =\sum_{j} r_{j}\left(t_{1}\right) \cdots r_{j}\left(t_{n-1}\right) x_{j}^{n}
\end{aligned}
$$

for $t_{1}, \ldots, t_{n-1} \in[0,1]$. Using the orthogonality of the Rademacher system and the Contraction Principle (see [21, page 231]) we have

$$
\begin{aligned}
& \sum_{j} A\left(\alpha_{j} x_{j}^{1}, \ldots, x_{j}^{n}\right) \\
& =\int_{0}^{1} \cdots \int_{0}^{1} A\left(f_{\alpha}\left(t_{1}\right), \ldots, f_{n-1}\left(t_{n-1}\right), f_{n}\left(t_{1}, \ldots, t_{n-1}\right)\right) d t_{1} \cdots d t_{n-1} \\
& \leq\|A\| \int_{0}^{1} \cdots \int_{0}^{1} \cdots\left(\int_{0}^{1}\left\|f_{\alpha}\left(t_{1}\right)\right\|\left\|f_{n}\left(t_{1}, \ldots, t_{n-1}\right)\right\| d t_{1}\right)\left\|f_{2}\left(t_{2}\right)\right\| \cdots\left\|f_{n-1}\left(t_{n-1}\right)\right\| d t_{2} \cdots d t_{n-1} \\
& \leq\|A\| \int_{0}^{1} \cdots \int_{0}^{1}\left\|\left(x_{j}^{1}\right)_{j}\right\|_{R a d_{2}}\left\|\left(x_{j}^{n}\right)_{j}\right\|_{R^{2} d_{2}}\left\|f_{2}\left(t_{2}\right)\right\| \cdots\left\|f_{n-1}\left(t_{n-1}\right)\right\| d t_{2} \cdots d t_{n-1}
\end{aligned}
$$

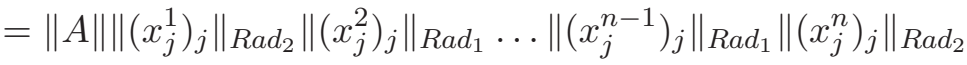

$$
\begin{aligned}
& \leq\|A\|\left\|\left(x_{j}^{1}\right)_{j}\right\|_{R a d} \cdots\left\|\left(x_{j}^{n}\right)_{j}\right\|_{R a d} \text {. }
\end{aligned}
$$

It is easy to see that $\|\hat{A}\| \geq\|A\|$ and we conclude the proof.

The following result, which appears in [33, Proposition 3.3], will be used several times in this paper (we include a short proof for the sake of completeness): 
Proposition 3.2 (Inclusion Theorem). Let $0<q \leq p \leq \infty, 0<q_{j} \leq p_{j} \leq \infty$ for all $j=1, \ldots, n$. If $\frac{1}{q_{1}}+\cdots+\frac{1}{q_{n}}-\frac{1}{q} \leq \frac{1}{p_{1}}+\cdots+\frac{1}{p_{n}}-\frac{1}{p}$ then

$$
\Pi_{\left(q ; q_{1}, \ldots, q_{n}\right)}\left(E_{1}, \ldots, E_{n} ; F\right) \subset \Pi_{\left(p ; p_{1}, \ldots, p_{n}\right)}\left(E_{1}, \ldots, E_{n} ; F\right)
$$

and $\pi_{\left(p ; p_{1}, \ldots, p_{n}\right)} \leq \pi_{\left(q ; q_{1}, \ldots, q_{n}\right)}$.

Proof. By the monotonicity of the $\ell_{p}$-norms we may assume $\frac{1}{q_{1}}+\cdots+\frac{1}{q_{n}}-\frac{1}{q}=\frac{1}{p_{1}}+\cdots+$ $\frac{1}{p_{n}}-\frac{1}{p}$. Let $A \in \Pi_{\left(q ; q_{1}, \ldots, q_{n}\right)}\left(E_{1}, \ldots, E_{n} ; F\right)$ and $\left(x_{j}^{k}\right)_{j=1}^{\infty} \in \ell_{p_{k}}^{w}\left(E_{k}\right), k=1, \ldots, n$, be given. We should prove that $\left(A\left(x_{j}^{1}, \ldots, x_{j}^{n}\right)\right)_{j=1}^{\infty} \in \ell_{p}(F)$, and for that it suffices to show that $\left(\alpha_{j} \cdot A\left(x_{j}^{1}, \ldots, x_{j}^{n}\right)\right)_{j=1}^{\infty} \in \ell_{q}(F)$ for every $\left(\alpha_{j}\right)_{j=1}^{\infty} \in \ell_{r}$ where $\frac{1}{p}+\frac{1}{r}=\frac{1}{q}$. Defining $r_{1}, \ldots, r_{n}$ by $\frac{1}{p_{j}}+\frac{1}{r_{j}}=\frac{1}{q_{j}}, j=1, \ldots, n$, it follows that $\frac{1}{r}=\frac{1}{r_{1}}+\cdots+\frac{1}{r_{n}}$. So $\ell_{r}=\ell_{r_{1}} \cdots \ell_{r_{n}}$. Given $\left(\alpha_{j}\right)_{j=1}^{\infty} \in \ell_{r}$, we write $\left(\alpha_{j}\right)_{j=1}^{\infty}=\left(\alpha_{j}^{1} \cdots \alpha_{j}^{n}\right)_{j=1}^{\infty}$ where $\left(\alpha_{j}^{k}\right)_{j=1}^{\infty} \in \ell_{r_{k}}, k=1, \ldots, n$. Since $\left(\alpha_{j}^{k}\right)_{j=1}^{\infty} \in \ell_{r_{k}}$ and $\left(x_{j}^{k}\right)_{j=1}^{\infty} \in \ell_{p_{k}}^{w}\left(E_{k}\right)$ it follows that $\left(\alpha_{j}^{k} x_{j}^{k}\right)_{j=1}^{\infty} \in \ell_{q_{k}}^{w}\left(E_{k}\right), k=1, \ldots, n$. Therefore

$$
\left(\alpha_{j} \cdot A\left(x_{j}^{1}, \ldots, x_{j}^{n}\right)\right)_{j=1}^{\infty}=\left(\alpha_{j}^{1} \cdots \alpha_{j}^{n} \cdot A\left(x_{j}^{1}, \ldots, x_{j}^{n}\right)\right)_{j=1}^{\infty}=\left(A\left(\alpha_{j}^{1} x_{j}^{1}, \ldots, \alpha_{j}^{n} x_{j}^{n}\right)\right)_{j=1}^{\infty} \in \ell_{q}(F)
$$

because $A$ is $\left(q ; q_{1}, \ldots, q_{n}\right)$-summing. The identifications and embeddings we used are all isometric, so the inequality between the norms follows.

Using Proposition 3.1 and the inclusion $\ell_{1}^{w}(E) \subset \operatorname{Rad}(E)$ one obtains Defant-Voigt's result. Now combining (9) with the inclusion theorem it is easy to prove that for $n \geq 2$ and $\frac{1}{p_{1}}+\cdots+\frac{1}{p_{n}} \geq \frac{1}{p}$ one has

$$
\mathcal{L}\left(E_{1}, \ldots, E_{n} ; \mathbb{K}\right)=\Pi_{\left(p ; p_{1}, \ldots, p_{n}\right)}\left(E_{1}, \ldots, E_{n} ; \mathbb{K}\right) \text { whenever } \frac{1}{p_{1}}+\cdots+\frac{1}{p_{n}}-\frac{1}{p} \geq n-1
$$

Before start exploring the inclusion theorem we show that sometimes the inclusion relationship turns out to be an equality. The next result is simple (it appeared in essence in [28, Theorem 16]) but indicates a good direction to be followed.

Proposition 3.3. Let $E_{1}, \ldots, E_{n}$ be cotype 2 spaces. Then

$$
\Pi_{\left(\frac{1}{n} ; 1, \ldots, 1\right)}\left(E_{1}, \ldots, E_{n} ; F\right)=\Pi_{\left(\frac{2}{n} ; 2, \ldots, 2\right)}\left(E_{1}, \ldots, E_{n} ; F\right)
$$

for every Banach space F.

Proof. It follows by combining (10) and the result saying that if $E_{j}$ has cotype 2 then $\Pi_{1}\left(E_{j} ; G_{j}\right)=\Pi_{2}\left(E_{j} ; G_{j}\right)$ (see [21, Corollary 11.16(a)]).

We aim to prove a more general result for cotype 2 spaces:

Theorem 3.4. Let $1 \leq k \leq n$ and assume that $E_{1}, \ldots, E_{k}$ have cotype 2. If $p \leq q$ and $1 \leq q_{i} \leq 2, i=1, \ldots, k$, satisfy that $\sum_{i=1}^{k} \frac{1}{q_{i}}-\frac{1}{q}=k-\frac{1}{p}$ then

$$
\Pi_{\left(p ; 1, \ldots, 1, p_{k+1}, \ldots, p_{n}\right)}\left(E_{1}, \ldots, E_{n} ; F\right)=\Pi_{\left(q ; q_{1}, \ldots, q_{k}, p_{k+1}, \ldots, p_{n}\right)}\left(E_{1}, \ldots, E_{n} ; F\right)
$$

for every Banach space $F$. 
Proof. The inclusion

$$
\Pi_{\left(p ; 1, \ldots, 1, p_{k+1}, \ldots, p_{n}\right)}\left(E_{1}, \ldots, E_{n} ; F\right) \subset \Pi_{\left(q ; q_{1}, \ldots, q_{k}, p_{k+1}, \ldots, p_{n}\right)}\left(E_{1}, \ldots, E_{n} ; F\right)
$$

follows from the Inclusion Theorem. Assume first that $q_{i}=2$ for $i=1, \ldots, k$ and $A \in$ $\Pi_{\left(q_{0} ; 2, \ldots, 2, p_{k+1}, \ldots, p_{n}\right)}\left(E_{1}, \ldots, E_{n} ; F\right)$ where $\frac{k}{2}+\frac{1}{q_{0}}=\frac{1}{p}$. Let $\left(x_{j}^{i}\right)_{j=1}^{\infty} \in \ell_{1}^{w}\left(E_{i}\right)$ for $i=1, \ldots, k$ and $\left(x_{j}^{i}\right)_{j=1}^{\infty} \in \ell_{p_{i}}^{w}\left(E_{i}\right)$ for $i=k+1, \ldots, n$. Since $E_{i}$ has cotype 2, by [3, Proposition 6(a)] we know that $\ell_{1}^{w}\left(E_{i}\right)=\ell_{2} \cdot \ell_{2}^{w}\left(E_{i}\right), i=1, \ldots, k$. Hence there are $\left(\alpha_{j}^{i}\right)_{j=1}^{\infty} \in \ell_{2}$ and $\left(y_{j}^{i}\right)_{j=1}^{\infty} \in$ $\ell_{2}^{w}\left(E_{k}\right)$ such that $\left(x_{j}^{i}\right)_{j=1}^{\infty}=\left(\alpha_{j}^{k} y_{j}^{i}\right)_{j=1}^{\infty}, i=1, \ldots, k$. In this fashion, $\left(\alpha_{j}^{1} \cdots \alpha_{j}^{k}\right)_{j=1}^{\infty} \in$ $\ell_{2} \cdots \ell_{2}=\ell_{\frac{2}{k}}$ and $\left(A\left(y_{j}^{1}, \ldots, y_{j}^{k}, x_{j}^{k+1}, \ldots, x_{j}^{n}\right)\right)_{j=1}^{\infty} \in \ell_{q_{0}}(F)$. Since $\frac{k}{2}+\frac{1}{q_{0}}=\frac{1}{p}$ it follows that

$$
\left(A\left(x_{j}^{1}, \ldots, x_{j}^{n}\right)\right)_{j=1}^{\infty}=\left(\alpha_{j}^{1} \cdots \alpha_{j}^{k} A\left(y_{j}^{1}, \ldots, y_{j}^{k}, x_{j}^{k+1}, \ldots, x_{j}^{n}\right)\right)_{j=1}^{\infty} \in \ell_{p}(F) .
$$

Now the general case follows again from the inclusion theorem, because the assumption gives that $\sum_{i=1}^{n} \frac{1}{q_{i}}-\frac{1}{q}=\frac{k}{2}-\frac{1}{q_{0}}$ and then

$$
\Pi_{\left(q ; q_{1}, \ldots, q_{k}, p_{k+1}, \ldots, p_{n}\right)}\left(E_{1}, \ldots, E_{n} ; F\right) \subset \Pi_{\left(q_{0} ; 2, \ldots, 2, p_{k+1}, \ldots, p_{n}\right)}\left(E_{1}, \ldots, E_{n} ; F\right) .
$$

Corollary 3.5. Let $1 \leq k \leq n$. Assume that $\mathcal{L}\left(E_{1}, \ldots, E_{k} ; F\right)=\Pi_{\left(p ; q_{1}, \ldots, q_{k}\right)}\left(E_{1}, \ldots, E_{k} ; F\right)$ and that $E_{k+1}, \ldots, E_{n}$ have cotype 2. If $p \leq q$ and $1 \leq q_{i} \leq 2, i=k+1, \ldots, n$, satisfy that $\sum_{i=k+1}^{n} \frac{1}{q_{i}}-\frac{1}{q}=n-k-\frac{1}{p}$ then

$$
\mathcal{L}\left(E_{1}, \ldots, E_{n} ; F\right)=\Pi_{\left(q ; q_{1}, \ldots, q_{n}\right)}\left(E_{1}, \ldots, E_{n} ; F\right) .
$$

Proof. From [12, Corollary 3.2] if $\mathcal{L}\left(E_{1}, \ldots, E_{k} ; F\right)=\Pi_{\left(p ; q_{1}, \ldots, q_{k}\right)}\left(E_{1}, \ldots, E_{k} ; F\right)$ then $\mathcal{L}\left(E_{1}, \ldots, E_{n} ; F\right)=\Pi_{\left(p ; q_{1}, \ldots, q_{k}, 1 \ldots, 1\right)}\left(E_{1}, \ldots, E_{n} ; F\right)$. An application of Theorem 3.4 yields the result.

\section{The role of the Littlewood-Orlicz property}

The aim of this section is to show how the Littlewood-Orlicz property can be used to obtain coincidence results stronger than (10). The proof of Theorem 4.1 will be also invoked in order to obtain new coincidence results for $n$-linear functionals on the disc algebra and on the Hardy space $\mathcal{H}^{\infty}$.

Theorem 4.1. Let $n \geq 2,1 \leq p_{i} \leq \infty, p_{n} \geq 2$ and

$$
n-\frac{3}{2} \leq \frac{1}{p_{1}}+\cdots+\frac{1}{p_{n}} .
$$

If $A \in \mathcal{L}\left(E_{1}, \ldots, E_{n} ; \mathbb{K}\right), E_{n}^{\prime}$ has the Littlewood-Orlicz property and

$$
n-\frac{3}{2} \leq \frac{1}{p_{1}}+\cdots+\frac{1}{p_{n}}-\frac{1}{p}
$$

then $\hat{A}: \ell_{p_{1}}^{w}\left(E_{1}\right) \times \cdots \times \ell_{p_{n}}^{w}\left(E_{n}\right) \rightarrow \ell_{p}$ is bounded. In other words, $\mathcal{L}\left(E_{1}, \ldots, E_{n} ; \mathbb{K}\right)=$ $\Pi_{\left(p ; p_{1}, \ldots, p_{n}\right)}\left(E_{1}, \ldots, E_{n} ; \mathbb{K}\right)$. Moreover $\|\hat{A}\| \leq\|A\|$. 
Proof. Denote by $A_{n-1}: E_{1} \times \cdots \times E_{n-1} \rightarrow E_{n}^{\prime}$ the corresponding $(n-1)$-linear mapping defined by

$$
A_{n-1}\left(x^{1}, \ldots, x^{n-1}\right)\left(x^{n}\right)=A\left(x^{1}, \ldots, x^{n-1}, x^{n}\right) .
$$

One has, using the previous results that

$$
\hat{A}_{n-1}: \ell_{1}^{w}\left(E_{1}\right) \times \cdots \times \ell_{1}^{w}\left(E_{n-1}\right) \rightarrow \ell_{1}^{w}\left(E_{n}^{\prime}\right)
$$

is bounded. In particular

$$
\hat{A}_{n-1}: \ell_{1}^{w}\left(E_{1}\right) \times \cdots \times \ell_{1}^{w}\left(E_{n-1}\right) \rightarrow \ell_{2} \otimes_{\pi} E_{n}^{\prime}
$$

is bounded. Now we use a duality argument. Note that

$$
\begin{gathered}
\hat{A}_{n-1}: \ell_{1}^{w}\left(E_{1}\right) \times \cdots \times \ell_{1}^{w}\left(E_{n-1}\right) \rightarrow \ell_{1}^{w}\left(E_{n}^{\prime}\right) \hookrightarrow \ell_{2} \otimes_{\pi} E_{n}^{\prime} \\
\hat{A}_{1}\left(\left(x_{j}^{1}\right)_{j}, \ldots,\left(x_{j}^{n-1}\right)_{j}\right)=\left(A\left(x_{j}^{1}, \ldots, x_{j}^{n-1}, \cdot\right)\right)_{j} \hookrightarrow \sum_{j=1}^{\infty} e_{j} \otimes A\left(x_{j}^{1}, \ldots, x_{j}^{n-1}, \cdot\right)
\end{gathered}
$$

is bounded. We have for some suitable $\varepsilon_{j}$ that

$$
\begin{aligned}
\left\|\widehat{A}\left(\left(x_{j}^{1}\right)_{j}, \ldots,\left(x_{j}^{n}\right)_{j}\right)\right\|_{1} & =\sum_{j}\left|A\left(x_{j}^{1}, \ldots, x_{j}^{n}\right)\right| \\
& =\left|\sum_{j} A\left(\varepsilon_{j} x_{j}^{1}, \ldots, x_{j}^{n}\right)\right| \\
& =\mid \sum_{j} A\left(\varepsilon_{j} x_{j}^{1}, \ldots, \frac{x_{j}^{n}}{\left\|\left(x_{j}^{n}\right)_{j}\right\|_{\ell_{2}^{w}\left(E_{n}\right)} \mid\left\|\left(x_{j}^{n}\right)_{j}\right\|_{\ell_{2}^{w}\left(E_{n}\right)}}\right. \\
& \leq{ }_{\left\|\left(y_{j}\right)_{j}\right\|_{\ell_{2}^{w}\left(E_{n}\right)} \leq 1,\left(y_{j}\right)_{j} \in \ell_{2}^{w}\left(E_{n}\right)}\left\{\left|\sum_{j} A\left(\varepsilon_{j} x_{j}^{1}, \ldots, y_{j}\right)\right|\left\|\left(x_{j}^{n}\right)_{j}\right\|_{\ell_{2}^{w}\left(E_{n}\right)}\right\} \\
& \leq \max _{\|u\| \leq 1, u \in \mathcal{L}\left(\ell_{2} ; E_{n}\right)}\left\{\left|\sum_{j} A\left(\varepsilon_{j} x_{j}^{1}, \ldots, u\left(e_{j}\right)\right)\right|\left\|\left(x_{j}^{n}\right)_{j}\right\|_{\ell_{2}^{w}\left(E_{n}\right)}\right\}=(*) .
\end{aligned}
$$

In this last inequality we used the identification:

$$
\begin{aligned}
\ell_{2}^{w}\left(E_{n}\right) & \longleftrightarrow \mathcal{L}\left(\ell_{2} ; E_{n}\right) \\
\left(y_{j}\right)_{j} & \longleftrightarrow T_{\left(y_{j}\right)_{j}}
\end{aligned}
$$

given by $T_{\left(y_{j}\right)_{j}}\left(\left(z_{j}\right)_{j}\right)=\sum_{j} y_{j} z_{j}$. Now, using the inclusion

$$
\begin{aligned}
\mathcal{L}\left(\ell_{2} ; E_{n}\right) & \hookrightarrow\left(\ell_{2} \otimes_{\pi} E_{n}^{\prime}\right)^{\prime} \\
u & \rightarrow \varphi: \ell_{2} \otimes_{\pi} E_{n}^{\prime} \rightarrow \mathbb{K} \\
\varphi\left(\left(\lambda_{j}\right)_{j} \otimes x^{\prime}\right) & =x^{\prime}\left(u\left(\left(\lambda_{j}\right)_{j}\right)\right)
\end{aligned}
$$

and the identification

$$
\begin{aligned}
\mathcal{L}\left(\ell_{2} ; E_{n}^{\prime \prime}\right) & =\left(\ell_{2} \otimes_{\pi} E_{n}^{\prime}\right)^{\prime} \\
S & \rightarrow \varphi_{S}: \ell_{2} \otimes_{\pi} E_{n}^{\prime} \rightarrow \mathbb{K} \\
\varphi_{S}(x \otimes y) & =S(x)(y)
\end{aligned}
$$


we get

$$
\begin{aligned}
& (*)=\max _{\|u\| \leq 1, u \in \mathcal{L}\left(\ell_{2} ; E_{n}\right)}\left\{\left|\sum_{j} A\left(\varepsilon_{j} x_{j}^{1}, \ldots, x_{j}^{n-1}, u\left(e_{j}\right)\right)\right|\left\|\left(x_{j}^{n}\right)_{j}\right\|_{\ell_{2}^{w}\left(E_{n}\right)}\right\} \\
& \leq \max _{\|\varphi\| \leq 1, \varphi \in\left(\ell_{2} \otimes_{\pi} E_{n}^{\prime}\right)^{\prime}}\left\{\left|\sum_{j} \varphi\left(e_{j} \otimes A\left(\varepsilon_{j} x_{j}^{1}, \ldots, x_{j}^{n-1}, \cdot\right)\right)\right|\left\|\left(x_{j}^{n}\right)_{j}\right\|_{\ell_{2}^{w}\left(E_{n}\right)}\right\} \\
& =\max _{\|\varphi\| \leq 1, \varphi \in\left(\ell_{2} \otimes_{\pi} E_{n}^{\prime}\right)^{\prime}}\left\{\left|\varphi\left(\sum_{j} e_{j} \otimes A\left(\varepsilon_{j} x_{j}^{1}, \ldots, x_{j}^{n-1}, \cdot\right)\right)\right|\left\|\left(x_{j}^{n}\right)_{j}\right\|_{\ell_{2}^{w}\left(E_{n}\right)}\right\} \\
& =\left\|\sum_{j} e_{j} \otimes A\left(\varepsilon_{j} x_{j}^{1}, \ldots, x_{j}^{n-1}, \cdot\right)\right\|_{\ell_{2} \otimes_{\pi} E_{n}^{\prime}}\left\|\left(x_{j}^{n}\right)_{j}\right\|_{\ell_{2}^{w}\left(E_{n}\right)} \\
& \stackrel{(* *)}{\leq} C\left\|\left(A\left(\varepsilon_{j} x_{j}^{1}, \ldots, x_{j}^{n-1}, \cdot\right)\right)_{j}\right\|_{\ell_{1}^{w}\left(E_{n}^{\prime}\right)}\left\|\left(x_{j}^{n}\right)_{j}\right\|_{\ell_{2}^{w}\left(E_{n}\right)} \\
& =C\left\|\hat{A}_{1}\left(\left(\varepsilon_{j} x_{j}^{1}\right)_{j}, \ldots,\left(x_{j}^{n-1}\right)_{j}\right)\right\|_{\ell_{1}^{w}\left(E_{n}^{\prime}\right)}\left\|\left(x_{j}^{n}\right)_{j}\right\|_{\ell_{2}^{w}\left(E_{n}\right)} \\
& \leq C\left\|\hat{A}_{1}\right\|\left\|\left(x_{j}^{1}\right)_{j}\right\|_{\ell_{1}^{w}\left(E_{1}\right)} \cdots\left\|\left(x_{j}^{n-1}\right)_{j}\right\|_{\ell_{1}^{w}\left(E_{n-1}\right)}\left\|\left(x_{j}^{n}\right)_{j}\right\|_{\ell_{2}^{w}\left(E_{n}\right)}<\infty,
\end{aligned}
$$

where in $(* *)$ we used that the inclusion $\ell_{1}^{w}\left(E_{n}^{\prime}\right) \hookrightarrow \ell_{2} \otimes_{\pi} E_{n}^{\prime}$ is continuous. We have just proved that

$$
\hat{A}: \ell_{1}^{w}\left(E_{1}\right) \times \cdots \times \ell_{1}^{w}\left(E_{n-1}\right) \times \ell_{2}^{w}\left(E_{n}\right) \rightarrow \ell_{1}
$$

is bounded. The proof is completed by using the Inclusion Theorem.

Taking into account the inclusion $\operatorname{Rad}(E) \subset \ell_{2}^{w}(E)$, our aim is now to analyze when the result in Proposition 3.1 can be lifted to $\ell_{2}^{w}\left(E_{i}\right)$. In other words, when $\mathcal{L}\left(E_{1}, \ldots,, E_{n}\right)=$ $\Pi_{(1 ; 2, \ldots, 2)}\left(E_{1}, \ldots, E_{n}\right)$. In this direction D. Pérez-García proved the following result:

Theorem 4.2. 34, Corollary 2.5] Let $E_{1}, \ldots, E_{n}$ be $\mathcal{L}_{\infty}$-spaces. If $A: E_{1} \times \cdots \times E_{n} \longrightarrow \mathbb{K}$ is multilinear and bounded, then $\hat{A}: \ell_{2}^{w}\left(E_{1}\right) \times \cdots \times \ell_{2}^{w}\left(E_{n}\right) \longrightarrow \ell_{1}$ is also bounded. In other words, $\mathcal{L}\left(E_{1}, \ldots, E_{n} ; \mathbb{K}\right)=\Pi_{(1 ; 2, \ldots, 2)}\left(E_{1}, \ldots, E_{n} ; \mathbb{K}\right)$.

Applying the idea used in the proof of Theorem 4.1 we can reprove the result above and generalize it to a larger class of spaces. Recall that a bilinear form $A: E_{1} \times E_{2} \longrightarrow \mathbb{K}$ is 2-dominated if and only if it is absolutely $(1 ; 2,2)$-summing, i.e., if and only if $\hat{A}: \ell_{2}^{w}\left(E_{1}\right) \times$ $\ell_{2}^{w}\left(E_{2}\right) \longrightarrow \ell_{1}$ is bounded.

Theorem 4.3. Let $n \geq 2$ and $E_{1}, \ldots, E_{n}$ be Banach spaces such that $E_{3}^{\prime}, \ldots, E_{n}^{\prime}$ have the Littlewood-Orlicz property and every continuous bilinear form on $E_{1} \times E_{2}$ is 2-dominated. If $A: E_{1} \times \cdots \times E_{n} \longrightarrow \mathbb{K}$ is multilinear and bounded, then $\hat{A}: \ell_{2}^{w}\left(E_{1}\right) \times \cdots \times \ell_{2}^{w}\left(E_{n}\right) \longrightarrow \ell_{1}$ is also bounded. In other words, $\mathcal{L}\left(E_{1}, \ldots, E_{n} ; \mathbb{K}\right)=\Pi_{(1 ; 2, \ldots, 2)}\left(E_{1}, \ldots, E_{n} ; \mathbb{K}\right)$.

Proof. We proceed by induction on $n$. The case $n=2$ follows by assumption. Assume that the result holds for $n \geq 2$. Let $B: E_{1} \times \cdots \times E_{n} \longrightarrow \mathbb{K}$ be given. By the induction hypothesis $\hat{B}: \ell_{2}^{w}\left(E_{1}\right) \times \cdots \times \ell_{2}^{w}\left(E_{n}\right) \longrightarrow \ell_{1}$ is bounded. It follows that for every Banach space $F$ and every $C: E_{1} \times \cdots \times E_{n} \longrightarrow F$, the mapping $\hat{C}: \ell_{2}^{w}\left(E_{1}\right) \times \cdots \times \ell_{2}^{w}\left(E_{n}\right) \longrightarrow \ell_{1}^{w}(F)$ is bounded. Given $A: E_{1} \times \cdots \times E_{n+1} \longrightarrow \mathbb{K}$, defining $A_{n}: E_{1} \times \cdots \times E_{n} \longrightarrow E_{n+1}^{\prime}$ in the 
obvious way, we have that $\hat{A}_{n}: \ell_{2}^{w}\left(E_{1}\right) \times \cdots \times \ell_{2}^{w}\left(E_{n}\right) \longrightarrow \ell_{1}^{w}\left(E_{n+1}^{\prime}\right)$ is bounded. Since $E_{n+1}^{\prime}$ has the Littlewood-Orlicz property we have that $\hat{A}_{n}: \ell_{2}^{w}\left(E_{1}\right) \times \cdots \times \ell_{2}^{w}\left(E_{n}\right) \longrightarrow \ell_{2} \otimes_{\pi} E_{n+1}^{\prime}$ is bounded. Using the duality argument from the proof of Theorem 4.1 it follows that $\hat{A}: \ell_{2}^{w}\left(E_{1}\right) \times \cdots \times \ell_{2}^{w}\left(E_{n+1}\right) \longrightarrow \ell_{1}$ is bounded as well.

Theorem 4.4. Let $E_{1}, \ldots, E_{n}$ be Banach spaces such that $E_{1}=E_{2}$ and each $E_{j}$ is either an $\mathcal{L}_{\infty}$-space, the disc algebra $\mathcal{A}$ or the Hardy space $\mathcal{H}^{\infty}$. Then $\mathcal{L}\left(E_{1}, \ldots, E_{n} ; \mathbb{K}\right)=$ $\Pi_{(1 ; 2, \ldots, 2)}\left(E_{1}, \ldots, E_{n} ; \mathbb{K}\right)$.

Proof. First we need to know that the duals of an $\mathcal{L}_{\infty}$-space, the disc algebra $\mathcal{A}$ and the Hardy space $\mathcal{H}^{\infty}$ have the Littlewood-Orlicz property. (i) It is well known that the dual of a $\mathcal{L}_{\infty}$-space has the Littlewood-Orlicz property. (ii) The dual $\mathcal{A}^{\prime}$ of the disc algebra is a G.T. space [14, Corollary 2.7] and has cotype 2 [14, Corollary 2.11], hence $\mathcal{A}^{\prime}$ has the Littlewood-Orlicz property. (iii) From [37, Theorem 6.17] (and using the notation from [37]) we know that $L_{1} / \overline{H_{0}^{1}}$ is a GT space of cotype 2. From [37, Proposition 6.2] we know that $\left(L_{1} / \overline{H_{0}^{1}}\right)^{\prime \prime}$ is a GT space. Since

$$
\mathcal{H}^{\infty}=\left(L_{1} / \overline{H_{0}^{1}}\right)^{\prime} \text { [37, Remark, page 84], }
$$

it follows that $\left(\mathcal{H}^{\infty}\right)^{\prime}$ is a GT space. It is well known that a Banach space has the same cotype of its bidual. So,

$$
\left(\mathcal{H}^{\infty}\right)^{\prime}=\left(L_{1} / \overline{H_{0}^{1}}\right)^{\prime \prime}
$$

has cotype 2. It follows that $\left(\mathcal{H}^{\infty}\right)^{\prime}$ has the Littlewood-Orlicz property. The fact that bilinear forms on either an $\mathcal{L}_{\infty}$-space or the disc algebra or the Hardy space are 2-dominated was proved in [7, Theorem 3.3] and [11, Proposition 2.1], respectively.

The same reasoning gives the following result:

Proposition 4.5. If $E_{2}^{\prime}, \ldots, E_{n}^{\prime}$ have the Littlewood-Orlicz property and $A: E_{1} \times \cdots \times$ $E_{n} \longrightarrow \mathbb{K}$ is multilinear and bounded, then $\mathcal{L}\left(E_{1}, \ldots, E_{n} ; \mathbb{K}\right)=\Pi_{(1 ; 1,2, \ldots, 2)}\left(E_{1}, \ldots, E_{n} ; \mathbb{K}\right)$.

\section{$5 \quad$ From bilinear to multilinear mappings}

In the previous section, when $E$ is an $\mathcal{L}_{\infty}$-space, the disc algebra $\mathcal{A}$ or the Hardy space $\mathcal{H}^{\infty}$, using that $\mathcal{L}\left({ }^{2} E ; \mathbb{K}\right)=\Pi_{(1 ; 2,2)}\left({ }^{2} E ; \mathbb{K}\right)$, we have shown that

$$
\mathcal{L}\left({ }^{n} E ; \mathbb{K}\right)=\Pi_{(1 ; 2, \ldots, 2)}\left({ }^{n} E ; \mathbb{K}\right)
$$

for every $n>2$. Although the lift of bilinear results to multilinear results is not a straightforward step in general, in the present section we obtain a general argument showing how bilinear coincidences of the type $\mathcal{L}\left({ }^{2} E ; \mathbb{K}\right)=\Pi_{(1 ; r, r)}\left({ }^{2} E ; \mathbb{K}\right)$ can generate coincidences for $n$-linear forms, $n \geq 3$.

Definition 5.1. Let $\frac{1}{q_{1}}+\frac{1}{q_{2}} \cdots+\frac{1}{q_{n}} \geq \frac{1}{p}$. We say that $A \in \mathcal{L}\left(E_{1}, \ldots, E_{n} ; F\right)$ is weakly $\left(p ; q_{1}, \ldots, q_{n}\right)$-summing if $\left(A\left(x_{j}^{1}, \ldots, x_{j}^{n}\right)\right)_{j} \in \ell_{p}^{w}(F)$ whenever $\left(x_{j}^{k}\right)_{j} \in \ell_{p}^{w}\left(E_{k}\right), k=1, \ldots, n$. The space formed by these mappings is denoted by $\Pi_{w\left(p ; q_{1}, \ldots, q_{n}\right)}\left(E_{1}, \ldots, E_{n} ; F\right)$ and the norm $\pi_{w\left(p ; q_{1}, \ldots, q_{n}\right)}$ is defined in the natural way.

Next Lemma is simple but useful: 
Lemma 5.2. Let $n \in \mathbb{N}$ and let $E_{1}, \ldots, E_{n}$ be Banach spaces. The following are equivalent:

(i) $\mathcal{L}\left(E_{1}, \ldots, E_{n} ; \mathbb{K}\right)=\Pi_{\left(p ; q_{1}, \ldots, q_{n}\right)}\left(E_{1}, \ldots, E_{n} ; \mathbb{K}\right)$ and $\pi_{\left(p ; q_{1}, \ldots, q_{n}\right)} \leq C\|\cdot\|$.

(ii) $\mathcal{L}\left(E_{1}, \ldots, E_{n} ; F\right)=\Pi_{w\left(p ; q_{1}, \ldots, q_{n}\right)}\left(E_{1}, \ldots, E_{n} ; F\right)$ for every Banach space $F$ and $\pi_{w\left(p ; q_{1}, \ldots, q_{n}\right)} \leq C\|\cdot\|$.

(iii) There exists $C>0$ such that

$$
\left\|\left(x_{j}^{1} \otimes \cdots \otimes x_{j}^{n}\right)_{j}\right\|_{\ell_{p}^{w}\left(E_{1} \otimes_{\pi} \cdots \otimes_{\pi} E_{n}\right)} \leq C \prod_{i=1}^{n}\left\|\left(x_{j}^{i}\right)_{j}\right\|_{\ell_{q_{i}}^{w}\left(E_{i}\right)}
$$

for all $\left(x_{j}^{i}\right)_{j} \in \ell_{q_{i}}^{w}\left(E_{i}\right), i=1, \ldots, n$.

Proof. $(i) \Longrightarrow(i i)$ Let $A \in \mathcal{L}\left(E_{1}, \ldots, E_{n} ; F\right)$. If $\left(x_{j}^{k}\right)_{j} \in \ell_{q_{k}}^{w}\left(E_{k}\right), k=1, \ldots, n$, then

$$
\begin{aligned}
\sup _{\varphi \in B_{F^{\prime}}}\left(\sum_{j}\left|\varphi\left(A\left(x_{j}^{1}, \ldots, x_{j}^{n}\right)\right)\right|^{p}\right)^{1 / p} & \leq \sup _{\varphi \in B_{F^{\prime}}} \pi_{\left(p ; q_{1}, \ldots, q_{n}\right)}(\varphi \circ A)\left\|\left(x_{j}^{1}\right)_{j}\right\|_{\ell_{q_{1}}^{w}\left(E_{1}\right)} \cdots\left\|\left(x_{j}^{n}\right)_{j}\right\|_{\ell_{q_{n}}^{w}\left(E_{n}\right)} \\
& \leq \sup _{\varphi \in B_{F^{\prime}}} C\|\varphi \circ A\|\left\|\left(x_{j}^{1}\right)_{j}\right\|_{\ell_{q_{1}}^{w}\left(E_{1}\right)} \cdots\left\|\left(x_{j}^{n}\right)_{j}\right\|_{\ell_{q_{n}}^{w}\left(E_{n}\right)} \\
& \leq C\|A\|\left\|\left(x_{j}^{1}\right)_{j}\right\|_{\ell_{q_{1}}^{w}\left(E_{1}\right)} \cdots\left\|\left(x_{j}^{n}\right)_{j}\right\|_{\ell_{q_{n}}^{w}\left(E_{n}\right)} .
\end{aligned}
$$

(ii) $\Longrightarrow($ iii $)$ Take $F=E_{1} \otimes_{\pi} \cdots \otimes_{\pi} E_{n}$ and $A: E_{1} \times \cdots \times E_{n} \rightarrow E_{1} \otimes_{\pi} \cdots \otimes_{\pi} E_{n}$ given by $A\left(x_{1}, \ldots, x_{n}\right)=x_{1} \otimes \cdots \otimes x_{n}$.

(iii) $\Longrightarrow\left(\right.$ i) Given $A \in \mathcal{L}\left(E_{1}, \ldots, E_{n} ; \mathbb{K}\right)$, its linearization $T: E_{1} \otimes_{\pi} \cdots \otimes_{\pi} E_{n} \rightarrow \mathbb{K}$ is bounded and then $\tilde{T}: \ell_{p}^{w}\left(E_{1} \otimes_{\pi} \cdots \otimes_{\pi} E_{n}\right) \rightarrow \ell_{p}$ is bounded. Now

$$
\begin{aligned}
\left\|\left(A\left(x_{j}^{1}, \ldots, x_{j}^{n}\right)\right)_{j}\right\|_{p} & =\left\|\left(T\left(x_{j}^{1} \otimes \cdots \otimes x_{j}^{n}\right)\right)_{j}\right\|_{p} \\
& \leq\|T\|\left\|\left(x_{j}^{1} \otimes \cdots \otimes x_{j}^{n}\right)_{j}\right\|_{\ell_{p}^{w}\left(E_{1} \otimes_{\pi} \cdots \otimes_{\pi} E_{n}\right)} \\
& \leq C\|T\| \prod_{i=1}^{n}\left\|\left(x_{j}^{i}\right)_{j}\right\|_{\ell_{q_{i}}^{w}\left(E_{i}\right)} .
\end{aligned}
$$

Theorem 5.3. Let $1 \leq r \leq 2$. If $\mathcal{L}\left({ }^{2} E ; \mathbb{K}\right)=\Pi_{(1 ; r, r)}\left({ }^{2} E ; \mathbb{K}\right)$ and $\pi_{(1 ; r, r)} \leq C\|\cdot\|$, then

(i) For $n$ even, $\mathcal{L}\left({ }^{n} E ; \mathbb{K}\right)=\Pi_{(1 ; r, \ldots, r)}\left({ }^{n} E ; \mathbb{K}\right)$ and $\pi_{(1 ; r, \ldots, r)} \leq C^{n / 2}\|\cdot\|$.

(ii) For $n \geq 3$ and odd, $\mathcal{L}\left({ }^{n} E ; \mathbb{K}\right)=\Pi_{(r ; r, \ldots, r)}\left({ }^{n} E ; \mathbb{K}\right)$ and $\pi_{(r ; r, \ldots, r)} \leq C^{(n-1) / 2}\|\cdot\|$.

Proof. (i) Let $n=2 m, m \in \mathbb{N}$, and $A \in \mathcal{L}\left({ }^{2 m} E ; \mathbb{K}\right)$. Using the associativity of the projective norm $\pi$ it is easy to see that there is an $m$-linear mapping $B \in \mathcal{L}\left({ }^{m}\left(E \hat{\otimes}_{\pi} E\right) ; \mathbb{K}\right)$ such that

$$
B\left(x^{1} \otimes x^{2}, \ldots, x^{2 m-1} \otimes x^{2 m}\right)=A\left(x^{1}, x^{2}, \ldots, x^{2 m-1}, x^{2 m}\right) .
$$


Using Defant-Voigt Theorem and Lemma 5.2 we get

$$
\begin{aligned}
\sum_{j} & \left|A\left(x_{j}^{1}, \ldots, x_{j}^{2 m}\right)\right| \\
& =\sum_{j}\left|B\left(x_{j}^{1} \otimes x_{j}^{2}, \ldots, x_{j}^{2 m-1} \otimes x_{j}^{2 m}\right)\right| \\
& \leq \pi_{(1 ; 1, \ldots, 1)}(B)\left\|\left(x_{j}^{1} \otimes x_{j}^{2}\right)_{j}\right\|_{\ell_{1}^{w}\left(E \otimes_{\pi} E\right)} \cdots\left\|\left(x_{j}^{2 m-1} \otimes x_{j}^{2 m}\right)_{j}\right\|_{\ell_{1}^{w}\left(E \otimes_{\pi} E\right)} \\
& \leq\|B\|\left(C\left\|\left(x_{j}^{1}\right)_{j}\right\|_{\ell_{r}^{w}(E)}\left\|\left(x_{j}^{2}\right)_{j}\right\|_{\ell_{r}^{w}(E)}\right) \cdots\left(C\left\|\left(x_{j}^{2 m-1}\right)_{j}\right\|_{\ell_{r}^{w}(E)}\left\|\left(x_{j}^{2 m}\right)_{j}\right\|_{\ell_{r}^{w}(E)}\right) \\
& =C^{m}\|A\|\left\|\left(x_{j}^{1}\right)_{j}\right\|_{\ell_{r}^{w}(E)} \cdots\left\|\left(x_{j}^{2 m}\right)_{j}\right\|_{\ell_{r}^{w}(E)} .
\end{aligned}
$$

(ii) Let $n=2 m+1, m \in \mathbb{N}$, and $A \in \mathcal{L}\left({ }^{2 m+1} E ; \mathbb{K}\right)$. From (i) and [12, Corollary 3.2] we conclude that $A \in \Pi_{(1 ; r, \ldots, r, 1)}\left({ }^{2 m+1} E ; \mathbb{K}\right)$ and it is not difficult to check that $\pi_{(1 ; r, \ldots, r, 1)} \leq$ $C^{m}\|\cdot\|$. Using the Inclusion Theorem we conclude that $A \in \Pi_{(p ; r, \ldots, r, p)}\left({ }^{2 m+1} E ; \mathbb{K}\right)$ for any $1 \leq p<\infty$. The result is now finished.

Let us point out some connection of Littlewood-Orlicz property on $E^{\prime}$ and $\mathcal{L}\left({ }^{2} E ; \mathbb{K}\right)=$ $\Pi_{(1 ; r, r)}\left({ }^{2} E ; \mathbb{K}\right)$.

Proposition 5.4. Let $E$ be a Banach space. The following statements are equivalent.

(i) $E^{\prime}$ has the Littlewood-Orlicz property.

(ii) $\mathcal{L}(X, E ; \mathbb{K})=\Pi_{(1 ; 1,2)}(X, E ; \mathbb{K})$ for any Banach space $X$.

(iii) $\ell_{1}^{w}(X) \otimes_{\pi} \ell_{2}^{w}(E) \subset \ell_{1}^{w}\left(X \otimes_{\pi} E\right)$.

Proof. $(i) \Longrightarrow($ ii $)$ Let $A: X \times E \rightarrow \mathbb{K}$ be a bounded bilinear form and let $T_{A}: X \rightarrow E^{\prime}$ be the corresponding linear operator. Assume that $\left(x_{j}\right)_{j} \in \ell_{1}^{w}(X)$ and $\left(y_{j}\right)_{j} \in \ell_{2}^{w}(E)$.

$$
\begin{aligned}
\sum_{j}\left|A\left(x_{j}, y_{j}\right)\right| & =\sum_{j}\left|T_{A}\left(x_{j}\right)\left(y_{j}\right)\right| \\
& =\sup _{\left|\alpha_{j}\right|=1}\left|\sum_{j} T_{A}\left(x_{j}\right)\left(\alpha_{j} y_{j}\right)\right| \\
& \leq\left\|\left(T_{A}\left(x_{j}\right)\right)_{j}\right\|_{\ell_{2} \otimes_{\pi}\left(E^{\prime}\right)}\left\|\left(y_{j}\right)_{j}\right\|_{\ell_{2}^{w}(E)} \\
& \leq C\left\|\left(T_{A}\left(x_{j}\right)\right)_{j}\right\|_{\ell_{1}^{w}\left(E^{\prime}\right)}\left\|\left(y_{j}\right)_{j}\right\|_{\ell_{2}^{w}(E)} \\
& \leq C\|A\|\left\|\left(x_{j}\right)_{j}\right\|_{\ell_{1}^{w}(X)}\left\|\left(y_{j}\right)_{j}\right\|_{\ell_{2}^{w}(E)} .
\end{aligned}
$$

$($ ii $) \Longrightarrow(i)$ Let $\left(x_{j}^{\prime}\right)_{j} \in \ell_{1}^{w}\left(E^{\prime}\right)$ be given. Consider the bounded bilinear map $A: c_{0} \times E \rightarrow \mathbb{K}$ defined by the condition $A\left(e_{j}, x\right)=x_{j}^{\prime}(x)$ for $x \in E$. To show that $\left(x_{j}^{\prime}\right)_{j} \in \ell_{2} \otimes_{\pi}\left(E^{\prime}\right)$ it suffices to see that

$$
\sum_{j}\left|x_{j}^{\prime}\left(x_{j}\right)\right| \leq C\left\|\left(x_{j}\right)_{j}\right\|_{\ell_{2}^{w}(E)}
$$

and, using $X=c_{0}$ in the assumption, this follows using that

$$
\sum_{j}\left|x_{j}^{\prime}\left(x_{j}\right)\right|=\sum_{j}\left|A\left(e_{j}, x_{j}\right)\right| \leq\|A\|\left\|\left(e_{j}\right)_{j}\right\|_{\ell_{1}^{w}\left(c_{0}\right)}\left\|\left(x_{j}\right)_{j}\right\|_{\ell_{2}^{w}(E)} .
$$

$($ ii $) \Longleftrightarrow($ iii) It is a particular case in Lemma 5.2 .

The same idea used in the proof of Theorem 5.3 provides the following slight improvement: 
Theorem 5.5. Let $n$ be a positive integer. For $i=1, \ldots, 2 n+1$ let $E_{i}$ be a Banach space and $1 \leq r_{2 n+1} \leq r_{1}, \ldots, r_{2 n} \leq 2$. If

$$
\begin{gathered}
\mathcal{L}\left(E_{1}, E_{2} ; \mathbb{K}\right)=\Pi_{\left(1 ; r_{1}, r_{2}\right)}\left(E_{1}, E_{2} ; \mathbb{K}\right) \text { and } \pi_{\left(1 ; r_{1}, r_{2}\right)} \leq C_{2}\|\cdot\|, \\
\mathcal{L}\left(E_{3}, E_{4} ; \mathbb{K}\right)=\Pi_{\left(1 ; r_{3}, r_{4}\right)}\left(E_{3}, E_{4} ; \mathbb{K}\right) \text { and } \pi_{\left(1 ; r_{3}, r_{4}\right)} \leq C_{4}\|\cdot\|, \ldots \\
\mathcal{L}\left(E_{2 n-1}, E_{2 n} ; \mathbb{K}\right)=\Pi_{\left(1 ; r_{2 n-1}, r_{2 n}\right)}\left(E_{2 n-1}, E_{2 n} ; \mathbb{K}\right) \text { and } \pi_{\left(1 ; r_{2 n-1}, r_{2 n}\right)} \leq C_{2 n}\|\cdot\|,
\end{gathered}
$$

then

$$
\begin{aligned}
\mathcal{L}\left(E_{1}, \ldots, E_{2 n} ; \mathbb{K}\right) & =\Pi_{\left(1 ; r_{1}, \ldots, r_{2 n}\right)}\left(E_{1}, \ldots, E_{2 n} ; \mathbb{K}\right) \text { and } \pi_{\left(1 ; r_{1}, \ldots, r_{2 n}\right)} \leq C_{2} \cdots C_{2 n}\|\cdot\|, \\
\mathcal{L}\left(E_{1}, \ldots, E_{2 n+1} ; \mathbb{K}\right) & =\Pi_{\left(r_{2 n+1} ; r_{1}, \ldots, r_{2 n+1}\right)}\left(E_{1}, \ldots, E_{2 n+1} ; \mathbb{K}\right) \text { and } \pi_{\left(r_{2 n+1} ; r_{1}, \ldots, r_{2 n+1}\right)} \leq C_{2} \cdots C_{2 n}\|\cdot\| .
\end{aligned}
$$

\section{The role of almost summing mappings}

Let $n \geq 2, A \in \mathcal{L}\left(E_{1}, \ldots, E_{n} ; F\right)$ and $1 \leq k \leq n$. Recall that the $k$-linear mapping $A_{k}$ is defined by

$A_{k}: E_{1} \times \cdots \times E_{k} \rightarrow \mathcal{L}\left(E_{k+1}, \ldots, E_{n} ; F\right), A_{k}\left(x_{1}, \ldots, x_{k}\right)\left(x_{k+1}, \ldots, x_{n}\right)=A\left(x_{1}, \ldots, x_{n}\right)$.

We first mention several connections between absolutely summing and almost summing multilinear mappings. Clearly $\Pi_{a . s}\left(E_{1}, \ldots, E_{n} ; F\right)$ coincides with $\Pi_{(2 ; 2, \ldots 2)}\left(E_{1}, \ldots, E_{n} ; F\right)$ whenever $F$ is a Hilbert space because $\operatorname{Rad}(F)=\ell_{2}(F)$, and the corresponding inclusions hold whenever $F$ has type $p$ or cotype $q$.

In the linear case one has (see [21]) $\bigcup_{p>0} \Pi_{p}(E ; F) \subset \Pi_{a . s}(E ; F)$. Using this linear containment relationship and (10) - see also [8] - it is not difficult to see that this relationship also holds for $p$-dominated multilinear maps, i.e.

$$
\bigcup_{p>0} \Pi_{(p / n ; p \ldots, p)}\left(E_{1}, \ldots, E_{n} ; F\right) \subset \Pi_{a . s}\left(E_{1}, \ldots, E_{n} ; F\right) .
$$

Proposition 6.1. Let $A \in \mathcal{L}\left(E_{1}, \ldots, E_{n} ; \mathbb{K}\right)$ and $A_{n-1} \in \mathcal{L}\left(E_{1}, \ldots, E_{n-1} ; E_{n}^{\prime}\right)$.

(i) If $A \in \Pi_{(1 ; 2, \ldots, 2)}\left(E_{1}, \ldots, E_{n} ; \mathbb{K}\right)$ then $A_{n-1} \in \Pi_{a . s}\left(E_{1}, \ldots, E_{n-1} ; E_{n}^{\prime}\right)$

(ii) If $E_{n}^{\prime}$ is a GT-space of cotype 2 and $A_{n-1} \in \Pi_{a . s}\left(E_{1}, \ldots, E_{n-1} ; E_{n}^{\prime}\right)$ then $A \in$ $\Pi_{(1 ; 2, \ldots, 2)}\left(E_{1}, \ldots, E_{n} ; \mathbb{K}\right)$.

Proof. (i) Assume $A \in \Pi_{(1 ; 2, \ldots, 2)}\left(E_{1}, \ldots, E_{n} ; \mathbb{K}\right)$. Using that $\ell_{2} \otimes_{\pi} F \subset \operatorname{Rad}(F)$ one has

$$
\begin{aligned}
\left\|\left(A_{n-1}\left(x_{j}^{1}, \ldots, x_{j}^{n-1}\right)\right)_{j}\right\|_{R a d\left(E_{n}^{\prime}\right)} & \leq C\left\|\left(A_{n-1}\left(x_{j}^{1}, \ldots, x_{j}^{n-1}\right)\right)_{j}\right\|_{\ell_{2} \otimes_{\pi} E_{n}^{\prime}} \\
& =\sup _{\left\|\left(x_{j}^{n}\right)_{j}\right\|_{2}^{w}\left(E_{n}\right)}\left|\sum_{j} A_{n-1}\left(x_{j}^{1}, \ldots, x_{j}^{n-1}\right)\left(x_{j}^{n}\right)\right| \\
& \leq \pi_{(1 ; 2, \ldots, 2)}(A) \prod_{i=1}^{n-1}\left\|\left(x_{j}^{i}\right)_{j}\right\|_{\ell_{2}^{w}\left(E_{i}\right) .}
\end{aligned}
$$


(ii)Assume that $A_{n-1} \in \Pi_{a . s}\left(E_{1}, \ldots, E_{n-1} ; E_{n}^{\prime}\right)$. From (44) one has $\ell_{2} \otimes_{\pi} E_{n}^{\prime}=\operatorname{Rad}\left(E_{n}^{\prime}\right)$. Hence we obtain, for any $\left|\alpha_{j}\right|=1$,

$$
\begin{aligned}
\sum_{j} A\left(\alpha_{j} x_{j}^{1}, \ldots, x_{j}^{n}\right) & =\sum_{j} A_{n-1}\left(\alpha_{j} x_{j}^{1}, \ldots, x_{j}^{n-1}\right)\left(x_{j}^{n}\right) \\
& \leq\left\|\left(A_{n-1}\left(\alpha_{j} x_{j}^{1}, \ldots, x_{j}^{n-1}\right)\right)_{j}\right\|_{\ell_{2} \otimes_{\pi} E_{n}^{\prime}}\left\|\left(x_{j}^{n}\right)_{j}\right\|_{\ell_{2}^{w}\left(E_{n}\right)} \\
& \leq C\left\|\left(A_{n-1}\left(\alpha_{j} x_{j}^{1}, \ldots, x_{j}^{n-1}\right)\right)_{j}\right\|_{R a d\left(E_{n}^{\prime}\right)}\left\|\left(x_{j}^{n}\right)_{j}\right\|_{\ell_{2}^{w}\left(E_{n}\right)} \\
& \leq C\left\|A_{n-1}\right\|_{a . s} \prod_{i=1}^{n}\left\|\left(x_{j}^{i}\right)_{j}\right\|_{\ell_{2}^{w}\left(E_{i}\right)}
\end{aligned}
$$

Theorem 6.2. Let $1 \leq k<n$ and $A \in \mathcal{L}\left(E_{1}, \ldots, E_{n} ; \mathbb{K}\right)$ be such that

$$
A_{k} \in \Pi_{a . s}\left(E_{1}, \ldots, E_{k} ; \mathcal{L}\left(E_{k+1}, . ., E_{n} ; \mathbb{K}\right)\right) \text {. }
$$

Then,

$$
\hat{A}: \ell_{2}^{w}\left(E_{1}\right) \times \ldots \times \ell_{2}^{w}\left(E_{k}\right) \times \operatorname{Rad}\left(E_{k+1}\right) \times \cdots \times \operatorname{Rad}\left(E_{n}\right) \rightarrow \ell_{1}
$$

is bounded. Moreover $\|\hat{A}\| \leq\left\|A_{k}\right\|_{a . s}$.

Proof. Let $\left(x_{j}^{i}\right)_{j}$ be a finite sequence in $E_{i}$ for $i=1, \ldots, n$. Take a scalar sequence $\left(\alpha_{j}\right)_{j}$, denote $A_{k}^{j}=A_{k}\left(x_{j}^{1}, x_{j}^{2}, \ldots, x_{j}^{k}\right)$ and define

$$
\begin{gathered}
f_{\alpha}\left(t_{k}\right)=\sum_{j} \alpha_{j} A_{k}^{j} r_{j}\left(t_{k}\right) ; f_{i}\left(t_{i}\right)=\sum_{j} r_{j}\left(t_{i}\right) x_{j}^{i}, i=k+1, \ldots, n-1 ; \text { and } \\
f_{n}\left(t_{k}, \ldots, t_{n-1}\right)=\sum_{j} r_{j}\left(t_{k}\right) \cdots r_{j}\left(t_{n-1}\right) x_{j}^{n}, \quad t_{k}, \ldots, t_{n-1} \in[0,1] .
\end{gathered}
$$

The orthogonality of the Rademacher system shows that

$$
\begin{array}{rl}
\sum_{j} & A\left(\alpha_{j} x_{j}^{1}, \ldots, x_{j}^{n}\right) \\
= & \sum_{j} A_{k}\left(\alpha_{j} x_{j}^{1}, \ldots, x_{j}^{k}\right)\left(x_{j}^{k+1}, \cdots, x_{j}^{n}\right) \\
= & \sum_{j} \alpha_{j} A_{k}^{j}\left(x_{j}^{k+1}, \cdots, x_{j}^{n}\right) \\
= & \int_{0}^{1} \cdots \int_{0}^{1} f_{\alpha}\left(t_{k}\right)\left(f_{k+1}\left(t_{k+1}\right), \ldots, f_{n-1}\left(t_{n-1}\right), f_{n}\left(t_{k}, \ldots, t_{n-1}\right)\right) d t_{k} \cdots d t_{n-1} \\
\leq & \int_{0}^{1} \cdots \int_{0}^{1}\left(\int_{0}^{1}\left\|f_{\alpha}\left(t_{k}\right)\right\|\left\|f_{n}\left(t_{k}, \ldots, t_{n-1}\right)\right\| d t_{k}\right)\left\|f_{k+1}\left(t_{k+1}\right)\right\| \cdots\left\|f_{n-1}\left(t_{n-1}\right)\right\| d t_{k+1} \cdots d t_{n-1} \\
\leq & \left\|A_{k}\right\|_{a . s} \prod_{i=1}^{k}\left\|\left(x_{j}^{i}\right)_{j}\right\|_{\ell_{2}^{w}\left(E_{i}\right)} \\
& \cdot \int_{0}^{1} \cdots \int_{0}^{1}\left(\int_{0}^{1}\left\|f_{n}\left(t_{k}, \ldots, t_{n-1}\right)\right\|^{2} d t_{k}\right)^{1 / 2}\left\|f_{k+1}\left(t_{k+1}\right)\right\| \cdots\left\|f_{n-1}\left(t_{n-1}\right)\right\| d t_{k+1} \cdots d t_{n-1} \\
\leq & \left\|A_{k}\right\|_{a . s} \prod_{i=1}^{k}\left\|\left(x_{j}^{i}\right)_{j}\right\|_{\ell_{2}^{w}\left(E_{i}\right)}\left(\prod_{i=k+1}^{n-1}\left\|\left(x_{j}^{i}\right)_{j}\right\|_{\text {Rad }_{1}}\right)\left\|\left(x_{j}^{n}\right)_{j}\right\|_{R_{a d}} .
\end{array}
$$

This allows to conclude the proof. 
Let us see that Theorem 6.2 has nice consequences.

Theorem 6.3. If $1 \leq p \leq 2$ and $E_{2}^{\prime}$ has type 2 , then

$$
\mathcal{L}\left(\ell_{p}, E_{2} ; \mathbb{K}\right)=\Pi_{(p ; 2,1)}\left(\ell_{p}, E_{2} ; \mathbb{K}\right)=\Pi_{(2 p /(2+p) ; 1,1)}\left(\ell_{p}, E_{2} ; \mathbb{K}\right)=\Pi_{\left(r_{p} ; r_{p}, r_{p}\right)}\left(\ell_{p}, E_{2} ; \mathbb{K}\right)
$$

for every $1 \leq r_{p} \leq \frac{2 p}{3 p-2}$.

Proof. We only treat the case $\mathbb{K}=\mathbb{C}$. The case $\mathbb{K}=\mathbb{R}$ follows from a complexification argument (see [10, 33] for details).

Assume first that $p=1$. Let $A \in \mathcal{L}\left(\ell_{1}, E_{2} ; \mathbb{K}\right)$. Since $E_{2}^{\prime}$ has type 2 , it follows from [21, Theorem 12.10] that $A_{1} \in \Pi_{a . s}\left(\ell_{1} ; E_{2}^{\prime}\right)$. So, from the previous theorem it follows that

$$
\hat{A}: \ell_{2}^{w}\left(\ell_{1}\right) \times \ell_{1}^{w}\left(E_{2}\right) \rightarrow \ell_{1}
$$

is bounded. Hence $A \in \Pi_{(1 ; 2,1)}\left(\ell_{1}, E_{2} ; \mathbb{K}\right)$. On the other hand, from the inclusion theorem we know that $\mathcal{L}\left(\ell_{2}, E_{2} ; \mathbb{K}\right)=\Pi_{(2 ; 2,1)}\left(\ell_{2}, E_{2} ; \mathbb{K}\right)$.

Let now $1 \leq p \leq 2$ and $A \in \mathcal{L}\left(\ell_{p}, E_{2} ; \mathbb{K}\right)$. Fix $\left(y_{j}\right) \in \ell_{1}^{w}\left(E_{2}\right)$ and consider the linear mappings

$$
T^{(1)}: \ell_{2}^{w}\left(\ell_{2}\right) \rightarrow \ell_{2} \text { and } T^{(2)}: \ell_{2}^{w}\left(\ell_{1}\right) \rightarrow \ell_{1}
$$

given by

$$
T^{(k)}\left(\left(x_{j}\right)_{j}\right)=\left(A\left(x_{j}, y_{j}\right)\right)_{j} \text { for } k=1,2 .
$$

Clearly $T^{(1)}$ and $T^{(2)}$ are well-defined and continuous. Using that $\ell_{2}^{w}\left(\ell_{t}\right)=\mathcal{L}\left(\ell_{2} ; \ell_{t}\right)$ for $t=1,2$, [38, proof of the Theorem] gives that

$$
\ell_{2}^{w}\left(\ell_{p}\right) \subset\left(\ell_{2}^{w}\left(\ell_{2}\right), \ell_{2}^{w}\left(\ell_{1}\right)\right)_{\theta}
$$

for $\frac{\theta}{2}=1-\frac{1}{p}$. So the complex interpolation method implies that

$$
\begin{gathered}
T: \ell_{2}^{w}\left(\ell_{p}\right) \rightarrow \ell_{p} \\
T\left(\left(x_{j}\right)_{j}\right)=\left(A\left(x_{j}, y_{j}\right)\right)_{j}
\end{gathered}
$$

is continuous. It follows that $A \in \Pi_{(p ; 2,1)}\left(\ell_{p}, E_{2} ; \mathbb{K}\right)$. Since $E_{2}^{\prime}$ has type 2 , it follows from [21, page 220] that $E_{2}$ has cotype 2 . Now use Theorem 3.4 to obtain $\Pi_{(p ; 2,1)}\left(\ell_{p}, E_{2} ; \mathbb{K}\right)=$ $\Pi_{(2 p /(2+p) ; 1,1)}\left(\ell_{p}, E_{2} ; \mathbb{K}\right)$. Using the inclusion theorem once again one has

$$
\Pi_{\left(\frac{2 p}{2+p} ; 1,1\right)}\left(\ell_{p}, E_{2} ; \mathbb{K}\right) \subset \Pi_{\left(s_{p} ; s_{p}, s_{p}\right)}\left(\ell_{p}, E_{2} ; \mathbb{K}\right)
$$

for $2-\frac{2+p}{2 p}=\frac{1}{s_{p}}$, which gives us $s_{p}=\frac{2 p}{3 p-2}$. So, since $1 \leq s_{p} \leq 2$, from [24, Theorem 3] it follows that $\Pi_{\left(r_{p} ; r_{p}, r_{p}\right)}\left(\ell_{p}, E_{2} ; \mathbb{K}\right)=\mathcal{L}\left(\ell_{p}, E_{2} ; \mathbb{K}\right)$ whenever $1 \leq r_{p} \leq s_{p}$.

Corollary 6.4. If $1 \leq p \leq 2$ and $1<q \leq 2$ then

(i) $\mathcal{L}\left(\ell_{p}, \ell_{q} ; \mathbb{K}\right)=\Pi_{(p ; 2,1)}\left(\ell_{p}, \ell_{q} ; \mathbb{K}\right)$

(ii) $\mathcal{L}\left(\ell_{1}, \ell_{q} ; \mathbb{K}\right)=\Pi_{(r ; r, r)}\left(\ell_{1}, \ell_{q} ; \mathbb{K}\right)$ for $1 \leq r \leq 2$.

The following result (for $n$-linear mappings) can also be obtained using results from [12] and the idea of the proof of Theorem 6.3.

Proposition 6.5. Let $n \geq 2$ and $1<p \leq 2$. Then every $n$-linear mapping $A \in \mathcal{L}\left(\ell_{1}, \stackrel{n-1}{\cdots}, \ell_{1}, \ell_{p} ; \mathbb{K}\right)$ is $\left(r_{n} ; r_{n}, \ldots, r_{n}\right)$-summing for every $1 \leq r_{n} \leq \frac{2^{n-1}}{2^{n-1}-1}$. 
Proof. The case $n=2$ is proved in Corollary 6.4(ii). From [24, Theorem 3 and Remark 2] it suffices to prove the result for $r_{n}=\frac{2^{n-1}}{2^{n-1}-1}$.

Case $n=3$ and $\mathbb{K}=\mathbb{C}$ : Let $A \in \mathcal{L}\left(\ell_{1}, \ell_{1}, \ell_{p} ; \mathbb{K}\right)$. From Corollary 6.4(i) we know that

$$
\mathcal{L}\left(\ell_{1}, \ell_{p} ; \mathbb{K}\right)=\Pi_{(1 ; 2,1)}\left(\ell_{1}, \ell_{p} ; \mathbb{K}\right) .
$$

From (11) and [12, Corollary 3.2] we get

$$
\mathcal{L}\left(\ell_{1}, \ell_{1}, \ell_{p} ; \mathbb{K}\right)=\Pi_{(1 ; 2,1,1)}\left(\ell_{1}, \ell_{1}, \ell_{p} ; \mathbb{K}\right)=\Pi_{(1 ; 1,2,1)}\left(\ell_{1}, \ell_{1}, \ell_{p} ; \mathbb{K}\right)
$$

So,

$$
\widehat{A}: \ell_{2}^{u}\left(\ell_{1}\right) \times \ell_{1}^{u}\left(\ell_{1}\right) \times \ell_{1}^{u}\left(\ell_{p}\right) \rightarrow \ell_{1}
$$

is bounded. Combining now Corollary 6.4(ii) with [12, Corollary 3.2] we get that

$$
\widehat{A}: \ell_{1}^{u}\left(\ell_{1}\right) \times \ell_{2}^{u}\left(\ell_{1}\right) \times \ell_{2}^{u}\left(\ell_{p}\right) \rightarrow \ell_{2}
$$

is bounded. So, using complex interpolation for (12) and (13) we conclude that

$$
\widehat{A}: \ell_{4 / 3}^{u}\left(\ell_{1}\right) \times \ell_{4 / 3}^{u}\left(\ell_{1}\right) \times \ell_{4 / 3}^{u}\left(\ell_{p}\right) \rightarrow \ell_{4 / 3}
$$

is bounded (this use of interpolation is based on results of [20], which are closely related to the classical paper [25] - further details can be found in [24]).

Case $n=4$ and $\mathbb{K}=\mathbb{C}$ : From the case $n=3$ and [12, Corollary 3.2] we know that

$$
\mathcal{L}\left(\ell_{1}, \ell_{1}, \ell_{1}, \ell_{p} ; \mathbb{K}\right)=\Pi_{\left(\frac{4}{3} ; 1, \frac{4}{3}, \frac{4}{3}, \frac{4}{3}\right)}\left(\ell_{1}, \ell_{1}, \ell_{1}, \ell_{p} ; \mathbb{K}\right)
$$

Since $\frac{4}{3}<2$, Corollary $6.4(i)$ gives that $\mathcal{L}\left(\ell_{1}, \ell_{p} ; \mathbb{K}\right)=\Pi_{\left(1 ; \frac{4}{3}, 1\right)}\left(\ell_{1}, \ell_{p} ; \mathbb{K}\right)$. So [12, Corollary 3.2] implies

$$
\mathcal{L}\left(\ell_{1}, \ell_{1}, \ell_{1}, \ell_{p} ; \mathbb{K}\right)=\Pi_{\left(1 ; \frac{4}{3}, 1,1,1\right)}\left(\ell_{1}, \ell_{1}, \ell_{1}, \ell_{p} ; \mathbb{K}\right)
$$

Hence

$$
\begin{aligned}
& \widehat{A}: \ell_{1}^{u}\left(\ell_{1}\right) \times \ell_{\frac{4}{3}}^{u}\left(\ell_{1}\right) \times \ell_{\frac{4}{3}}^{u}\left(\ell_{1}\right) \times \ell_{\frac{4}{3}}^{u}\left(\ell_{p}\right) \rightarrow \ell_{\frac{4}{3}} \text { and } \\
& \widehat{A}: \ell_{\frac{4}{3}}^{u}\left(\ell_{1}\right) \times \ell_{1}^{u}\left(\ell_{1}\right) \times \ell_{1}^{u}\left(\ell_{1}\right) \times \ell_{1}^{u}\left(\ell_{p}\right) \rightarrow \ell_{1}
\end{aligned}
$$

are bounded. Using complex interpolation once more we conclude that

$$
\widehat{A}: \ell_{\frac{8}{7}}^{u}\left(\ell_{1}\right) \times \ell_{\frac{8}{7}}^{u}\left(\ell_{1}\right) \times \ell_{\frac{8}{7}}^{u}\left(\ell_{1}\right) \times \ell_{\frac{8}{7}}^{u}\left(\ell_{p}\right) \rightarrow \ell_{\frac{8}{7}}
$$

is bounded as well. The cases $n>4$ are similar and the real case follows by complexification.

\section{References}

[1] R. Alencar and M. C. Matos, Some classes of multilinear mappings between Banach spaces, Publicationes Universidad Complutense de Madrid, 12 (1989).

[2] R. Aron, M. Lacruz, R. Ryan and A. Tonge, The generalized Rademacher functions, Note Mat. 12 (1992), 15-25.

[3] J. L. Arregui, O. Blasco, (p, q)-Summing sequences, J. Math. Anal. Appl. 274 (2002), 812-827. 
[4] S. Aywa, J. H. Fourie, On summing multipliers and applications. J. Math. Anal. Appl. 253 (2001), 166-186.

[5] J. Bergh and J. Löfström, Interpolation spaces, Springer-Verlag, 1976.

[6] H. F. Bohnenblust and E. Hille, On the absolute convergence of Dirichlet series, Ann. of Math. (2) 32 (1931), 600-622.

[7] G. Botelho, Cotype and absolutely summing multilinear mappings and homogeneous polynomials, Proc. Roy. Irish Acad. Sect. A 97 (1997), 145-153.

[8] G. Botelho, Almost summing polynomials, Math. Nachr. 212 (2000), 25-36.

[9] G. Botelho, H.-A. Braunss, H. Junek, Almost p-summing polynomials and multilinear mappings, Arch. Math. 76 (2001), 109-118.

[10] G. Botelho, H.-A. Braunss, H. Junek and D. Pellegrino, Inclusions and coincidences for multiple summing multilinear mappings, Proc. Amer. Math. Soc., to appear.

[11] G. Botelho and D. Pellegrino, Scalar-valued dominated polynomials on Banach spaces, Proc. Amer. Math. Soc. 134 (2006), 1743-1751.

[12] G. Botelho and D. Pellegrino, Coincidence situations for absolutely summing non-linear mappings, Port. Math. 64 (2007), 176-191.

[13] G. Botelho, D. Pellegrino and P. Rueda, Summability and estimates for polynomials and multilinear mappings, Indag. Math., to appear.

[14] J. Bourgain, New Banach space properties of the disc algebra and $\mathcal{H}^{\infty}$, Acta Math. 152 (1984), 1-48.

[15] Q. Bu and J. Diestel, Observations about the projective tensor product of Banach spaces, I $\ell_{p} \hat{\otimes} X, 1<p<\infty$, Quaest. Math. 24 (2001), 519-533.

[16] Q. Bu, On Banach spaces verifying Grothendieck's Theorem, Bull. London Math. Soc. 35 (2003), $738-748$.

[17] Y. S. Choi, S. G. Kim, Y. Meléndez and A. Tonge, Estimates for absolutely summing norms of polynomials and multilinear maps, Q. J. Math. 52 (2001), 1-12.

[18] J. S. Cohen, Absolutely p-summing, p-nuclear operators and their conjugates. Math. Ann. 201 (1973), 177-200.

[19] A. Defant, K. Floret, Tensor Norms and Operator Ideals. North-Holland Publishing Co., Amsterdam, 1993.

[20] A. Defant and C. Michels, A complex interpolation formula for tensor products of vector-valued Banach function spaces, Arch. Math. 74 (2000), 441- 451.

[21] J. Diestel, H. Jarchow, A. Tonge, Absolutely summing operators. Cambridge University Press, 1995.

[22] S. Dineen, Complex Analysis on Infinite Dimensional Spaces, Springer Verlag, London, 1999.

[23] D. J. H. Garling, Inequalities: a journey into linear analysis, Cambridge University Press, 2007.

[24] H. Junek, M. Matos, D. Pellegrino, Inclusion theorems for absolutely summing holomorphic mappings, Proc. Amer. Math. Soc. 136 (2008), 3983-3991.

[25] O. Kouba, On interpolation of injective or projective tensor products of Banach spaces, J. Funct. Anal. 96 (1991), 38-61.

[26] J. Lindenstrauss and A. Pełczyński, Absolutely summing operators in $\mathcal{L}_{p}$-spaces and their applications, Studia Math. 29 (1968), 275-325. 
[27] J. Littlewood, On bounded bilinear forms in an infinite number of variables, Q. J. Math. 2 (1930), 167-171.

[28] Y. Meléndez and A. Tonge, Polynomials and the Pietsch Domination Theorem, Math. Proc. R. Ir. Acad 99A (1999), 195-212.

[29] J. Mujica, Complex Analysis in Banach Spaces, North-Holland Mathematics Studies, NorthHolland, 1986.

[30] D. Pellegrino, Cotype and absolutely summing homogeneous polynomials in $\mathcal{L}_{p}$ spaces, Studia Math. 157 (2003), 121-131.

[31] D. Pellegrino, Almost summing mappings, Arch. Math. 82 (2004), 68-80.

[32] D. Pellegrino, On scalar valued nonlinear absolutely summing mappings, Ann. Pol. Math. 83 (2004), 281-288.

[33] D. Pérez-García, Operadores multilineales absolutamente sumantes, Thesis, Universidad Complutense de Madrid, 2003.

[34] D. Pérez-García, The trace class is a Q-algebra, Ann. Acad. Sci. Fenn. Math. 21 (2006), $287-295$.

[35] D. Pérez-García, M. M. Wolf, C. Palazuelos, I. Villanueva, M. Junge, Unbounded violation of tripartite Bell inequalities, Commun. Math. Phys. 279 (2008), 455-486.

[36] A. Pietsch, Ideals of multilinear functionals (designs of a theory), Proceedings of the second international conference on operator algebras, ideals, and their applications in theoretical physics (Leipzig, 1983), 185-199, Teubner-Texte Math., 67, Teubner, Leipzig, 1984.

[37] G. Pisier, Factorization of Linear Operators and Geometry of Banach spaces. CBMS 60. Amer. Math. Soc. Providence R.I., 1986.

[38] G. Pisier, A remark on $\Pi_{2}\left(\ell^{p}, \ell^{p}\right)$. Math. Nachr. 148 (1990), 243-245.

[39] M. Talagrand, Cotype of operators from $C(K)$. Invent. Math. 107 (1992), 1-40.

[40] M. Talagrand, Cotype and $(1, q)$-summing norm in a Banach space. Invent. Math. 110 (1992), $545-556$.

[41] N. Tomczak-Jaegermann, Banach-Mazur distances and finite-dimensional Operator Ideals. Longman Scientific and Technical, 1989.

[42] N. N. Vakhania, V. I. Tarieladze, S. A. Chobanyan, Probability distributions on Banach spaces D. Reidel, Dordrecht, 1987.

[Oscar Blasco] Departamento de Análisis Matemático, Universidad de Valencia, 46.100 Burjasot - Valencia, Spain, e-mail: oscar.blasco@uv.es

[Geraldo Botelho] Faculdade de Matemática, Universidade Federal de Uberlândia, 38.400902 - Uberlândia, Brazil, e-mail: botelho@ufu.br

[Daniel Pellegrino] Departamento de Matemática, Universidade Federal da Paraíba, 58.051900 - João Pessoa, Brazil, e-mail: dmpellegrino@gmail.com

[Pilar Rueda] Departamento de Análisis Matemático, Universidad de Valencia, 46.100 Burjasot - Valencia, Spain, e-mail: pilar.rueda@uv.es 